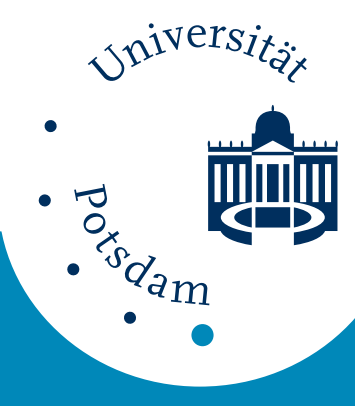

Universität Potsdam

Martin G. Peter, Peter C. Boldt, Yvonne Niederstein, Jasna Peter-Katalinić

\title{
Synthesen von Galactose-Cluster-haltigen Steroid-Derivaten
}

first published in:

Liebigs Annalen der Chemie - 9 (1990), p. 863 - 869

ISSN (print): 1434-193X

ISSN (online): 1099-0690

DOI: 10.1002/jlac.1990199001162

Postprint published at the institutional repository of Potsdam University:

In: Postprints der Universität Potsdam :

Mathematisch-Naturwissenschaftliche Reihe ; 42

http://opus.kobv.de/ubp/volltexte/2008/1678/

http://nbn-resolving.de/urn:nbn:de:kobv:517-opus-16783

Postprints der Universität Potsdam

Mathematisch-Naturwissenschaftliche Reihe ; 42 


\title{
Synthesen von Galactose-Cluster-haltigen Steroid-Derivaten ${ }^{\neq)}$
}

\author{
Martin G. Peter*a ${ }^{*}$ Peter C. Boldt ${ }^{\neq \neq) a}$, Yvonne Niederstein ${ }^{\neq \neq \neq) a}$ und Jasna Peter-Katalinić ${ }^{b}$ \\ Institut für Organische Chemie und Biochemie ${ }^{a}$ der Universität Bonn, \\ Gerhard-Domagk-Straße 1, D-5300 Bonn 1, F.R.G. \\ Institut für Physiologische Chemie ${ }^{\mathbf{b}}$ der Universität Bonn, \\ Nußallee 11, D-5300 Bonn, F.R.G.
}

Eingegangen am 23. März 1990

Key Words: Glycoconjugates / Galactosides / Steroid esters / Amphiphiles / Glycopeptides

\section{Synthesis of Galactose-Cluster-Containing Steroid Derivatives}

The synthesis of galactose clusters that are linked to a steroid moiety by a peptide-like spacer unit is described. The galactose cluster is obtained by Koenigs-Knorr glycosylation of TRIS-Gly-Fmoc (2b) under Helferich conditions. Peptide and ester bonds are formed after activation of carboxylic acids as diphenylthiophene dioxide (TDO) esters. $6 \mathbf{a}$ is synthesized in a convergent way by coupling of $\left(\mathrm{Ac}_{4} \mathrm{Gal}\right)_{3}$-TRIS-Gly (3e) with cholesteryl TDO succinate (5b). Coupling of $\left(\mathrm{Ac}_{4} \mathrm{Gal}\right)_{3}$-TRISGly hydrogen succinate (3f) with Gly-O-Chol (5d) by means of EEDQ yields $6 \mathbf{d}$. Reaction of $\left(\mathrm{Ac}_{4} \mathrm{Gal}\right)_{3}$-TRIS-Gly-SUCC-OTDO (3g) with 25-hydroxycholesterol leads in a linear sequence to the oxysterol derivative $6 \mathbf{f}$. Selective cleavage of the acetyl groups from galactose units yields the known compound $6 \mathrm{~b}$ and the new derivatives $6 \mathbf{e}$ and $6 \mathrm{~g}$.
Neoglycokonjugate sind synthetische Analoga von natürlich vorkommenden Glycolipiden und Glycoproteinen. Als charakteristische Strukturmerkmale enthalten sie glycosidisch gebundene Zukker-Reste, die von spezifischen, auf der Außenseite der Plasmamembran von Zellen lokalisierten Rezeptoren erkannt werden ${ }^{1)}$. In letzter Zeit beanspruchen solche Neoglycokonjugate besonderes Interesse, bei denen Rezeptor-affine Kohlenhydrate mit Wirkstoffen verknüpft sind. Die Internalisierung des Ligand-Rezeptor-Komplexes läßt sich zum zellspezifischen Drug-Targeting und zur intrazellulären Freisetzung von Pharmakophoren ausnutzen. So gelingt es, in mannosylierte Liposomen verpackte Wirkstoffe gezielt in Makrophagen einzuschleusen ${ }^{2}$. Galactosyliertes und mit Acyclovir verknüpftes Polylysin ${ }^{3)}$, galactosylierte Liposomen ${ }^{4)}$, lactosylierte Lipoproteine $^{5)}$ oder Asialoglycoproteine werden dagegen hauptsächlich über einen Galactose/ $N$-Acetylgalactosamin-spezifischen Rezeptor, den sog. Asialoprotein- oder Ashwell-Rezeptor ${ }^{1,6)}$, in Leberzellen aufgenommen.

Detaillierte Studien von Lee et al. ${ }^{7)}$ zur Liganden-Spezifität dieses Rezeptors zeigen, daß die Bindungsstellen für Galactose an den Spitzen eines Dreiecks mit den Seitenlängen 1.5, 2.2 und $2.5 \mathrm{~nm}$ lokalisiert sind. Infolgedessen beobachtet man hohe Bindungsaffinität für Verbindungen, die Kohlenhydrat-Cluster aus mindestens drei Galactose-Einheiten enthalten und somit die Strukturmerkmale von tri-antennären $N$-Glycanen der Asialoproteine tragen. Im Gegensatz dazu besitzt das Galactose-Erkennungssystem von Makrophagen höchste Affinität für tetra-antennäre Asialo- $N$-GlycanStrukturen ${ }^{8)}$.

\footnotetext{
Abkürzungen

$\mathrm{Ac}_{4} \mathrm{Gal}:$ 2,3,4,6-Tetra- $O$-acetyl-D-galactopyranosyl-; Chol: Cholesterin; EEDQ: 2-Ethoxy-1-(ethoxycarbonyl)-1,2-dihydrochinolin; Fmoc: (9-Fluorenyl)methoxycarbonyl-; Gal: D-Galactopyranosyl-; SUCC: Succinyl-; TDO: 1,1,3-Trioxo-2,5-diphenyl-2,3-dihydrothiophen-4-yl-; TRIS: 2-Amino-2-(hydroxymethyl)-1,3-propandiol.

\#) Auszugweise vorgetragen auf der Chemiedozenten-Tagung Bielefeld, 7. März 1989.

++) Teil der Diplomarbeit, Universität Bonn 1988.

$+\neq \neq)$ Teil der geplanten Dissertation, Universität Bonn.
}

Kempen et al. ${ }^{97}$ beschrieben kürzlich das amphiphile CholesterinDerivat $\mathbf{6 b}$, bei dem der lipophile Rest über einen Peptid-ähnlichen Spacer mit einem Cluster von drei Galactose-Einheiten verknüpft ist. Infusion von $\mathbf{6 b}$ in Ratten führt zu einer Senkung des Cholesterin-Spiegels ${ }^{10)}$, die letztlich auf die feed-back-Hemmung der Cholesterin-Biosynthese aufgrund der vermehrten Einschleusung von Lipoproteinen in Leberzellen über den Asialoprotein-Rezeptor zurückgeführt werden kann ${ }^{10,11)}$.

Verbindungen des Typs 6 eignen sich nicht nur zur Untersuchung von zellbiologischen Phänomenen. Die Herstellung von glycosidischen Clustern und deren Verknüpfung mit Wirkstoffen bietet auch eine reizvolle präparative Aufgabe. Vor diesem Hintergrund berichten wir nun über eine neue Synthese von 6b sowie über die Herstellung der bisher nicht beschriebenen Galactose-Cluster-haltigen Steroid-Derivate 6e und 6g. Letztere unterscheiden sich von 6b durch Variation des Peptid-ähnlichen Spacers bzw. der SteroidEinheit. Laut Molekülmodell-Berechnungen des Galactose-TRISClusters ${ }^{12)}$ lassen sich die für die Rezeptor-Spezifität primär verantwortlichen OH-Gruppen an C-4 der Galactose-Reste ${ }^{8 i}$ an den Spitzen eines Dreiecks mit den Seitenlängen 1.01, 0.91 und $0.84 \mathrm{~nm}$ anordnen. Diese Abstände sind etwa nur halb so groß wie die von Lee et al. ${ }^{7}$ postulierten optimalen Werte; da $\mathbf{6} \mathbf{b}$ jedoch biologische Aktivität in vivo zeigt ${ }^{10,11}$, haben wir die Kohlenhydrat-Kopfgruppe vorerst nicht variiert.

Zur Synthese von Galactose-Clustern des Typs 6 ist es zunächst erforderlich, ein TRIS-Derivat möglichst vollständig zu galactosidieren. Gemäß Literturangaben ${ }^{9)}$ entsteht bei der Helferich-Variante der Koenigs-Knorr-Reaktion ${ }^{13)}$ von TRIS-Gly-Z (2a) mit Acetobromgalactose (Schema 1) in 32 proz. Ausbeute eine Mischung von $\left(\mathrm{Ac}_{4} \mathrm{Gal}\right)_{3}$-TRISGly-Z (3a) und des bis-galactosylierten $\left(\mathrm{Ac}_{4} \mathrm{Gal}\right)_{2}$-TRIS-GlyZ (3b) im Verhältnis ca. 5:1 (vgl. Lit. ${ }^{14)}$ ). Nach unseren Erfahrungen liefert die Umsetzung von Acetobromgalactose mit $\mathbf{2 a}$ das gewünschte $\mathbf{3 a}$ in nur geringer Ausbeute.

Wesentliche Verbesserungen ergeben sich bei Verwendung der Fmoc-Schutzgruppe. TRIS-Gly-Fmoc (2 b) wird in 
Schema 1

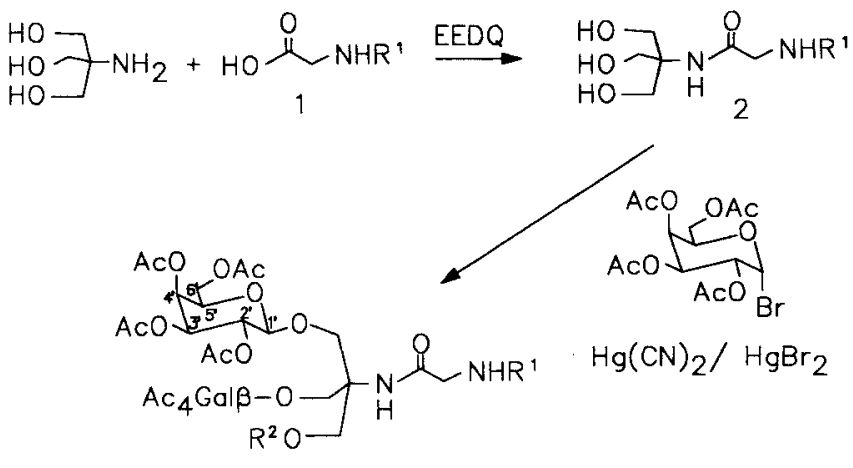

3

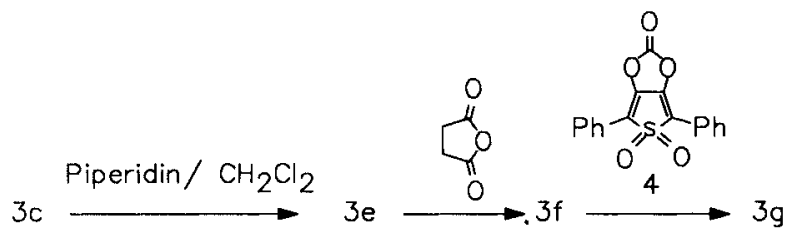

\begin{tabular}{l|ll} 
& $\mathrm{R}^{1}$ & $\mathrm{R}^{2}$ \\
\hline $\mathbf{3 a}$ & $\mathrm{Z}$ & $\mathrm{Ac}_{4} \mathrm{Gal}$ \\
$\mathbf{3 b}$ & $\mathrm{Z}$ & $\mathrm{OH}$ \\
$\mathbf{3 c}$ & $\mathrm{Fmoc}$ & $\mathrm{Ac}$ Gal \\
$\mathbf{3 d}$ & $\mathrm{Fmoc}$ & $\mathrm{Ac}$ \\
$\mathbf{3 e}$ & $\mathrm{H}$ & $\mathrm{Ac}_{4} \mathrm{Gal}$ \\
$\mathbf{3 f}$ & $\mathrm{COCH}_{2} \mathrm{CH}_{2} \mathrm{CO}_{2} \mathrm{H}$ & $\mathrm{Ac}_{4} \mathrm{Gal}$ \\
$\mathbf{3 g}$ & $\mathrm{COCH}_{2} \mathrm{CH}_{2} \mathrm{CO}_{2} \mathrm{TDO}$ & $\mathrm{Ac}_{4} \mathrm{Gal}$
\end{tabular}

55 proz. Ausbeute hergestellt. Die nachfolgende Glycosidierung mit Acetobromgalactose ${ }^{13\}}$ liefert ein gemä $B$ DC einheitliches Produkt der Summenformel $\mathrm{C}_{63} \mathrm{H}_{78} \mathrm{~N}_{2} \mathrm{O}_{33} \cdot 2.5$ $\mathrm{H}_{2} \mathrm{O}$ in 38 proz. Ausbeute. Die ${ }^{1} \mathrm{H}$ - und ${ }^{13} \mathrm{C}-\mathrm{NMR}$-Spektren zeigen die für $3 c$ erwarteten Signale (siehe Exp. Teil). Die $\beta$ Konfiguration der glycosidischen Bindung folgt im ${ }^{1} \mathrm{H}$ NMR Spektrum aus der Kopplungskonstanten von 1'-H $(J=7.7 \mathrm{~Hz})$ und im ${ }^{13} \mathrm{C}$-NMR Spektrum aus der Verschiebung von $\mathrm{C}-1^{\prime}(\delta=101.16)$ der Galactosyl-Reste. Das FABMassenspektrum liefert die Nominalmasse $m / z=1413$ $\left[\mathrm{M}+\mathrm{Na}^{+}\right]$bzw. $1391\left[\mathrm{M}+\mathrm{H}^{+}\right]$, sowie die nach $\mathrm{Ab}-$ spaltung von Tetraacetylgalactose bzw. Keten entstandenen Fragment-Ionen $m / z=1043$ bzw. 1371 und 1001 (Abb. 1). Daneben deutet jedoch die Serie der Peaks bei $m / z=1125$ und 755 sowie 1083, 1061 und 713 auf die Anwesenheit des zweifach glycosidierten Acetats 3d (Abb. 1) hin. Somit beweist das Massenspektrum, daß bei der Umsetzung von $\mathbf{2 b}$ mit Acetobromgalactose eine primäre Hydroxy-Gruppe des TRIS-Rests partiell acetyliert wird. Vermutlich reagiert hierbei das aus Acetobromgalactose unter Nachbargruppenbeteiligung entstehende Acyloxonium-Ion unter Bildung des Acetats (vgl. Lit. ${ }^{15)}$ ). Anhand der ${ }^{1} \mathrm{H}$ - und ${ }^{13} \mathrm{C}-\mathrm{NMR}$-Spektren und der Elementaranalyse läßt sich abschätzen, daß der relative Anteil von 3d weniger als $10 \%$ beträgt. Die Umsetzung von $\mathbf{2} \mathbf{b}$ mit Pentaacetylgalactose in Gegenwart von TMS-Triflat führt ebenfalls zur Mischung von $\mathbf{3 c}$ und $\mathbf{3 d}$, wobei die Produktzusammensetzung jedoch wesentlich höhere Anteile von 3d aufweist (MS-Evidenz). Nach chromatographischer Trennung von 3c und 3d an Kieselgel behandelt man 3c mit Piperidin. Das freie Amin 3e wird in 93 proz. Ausbeute isoliert.
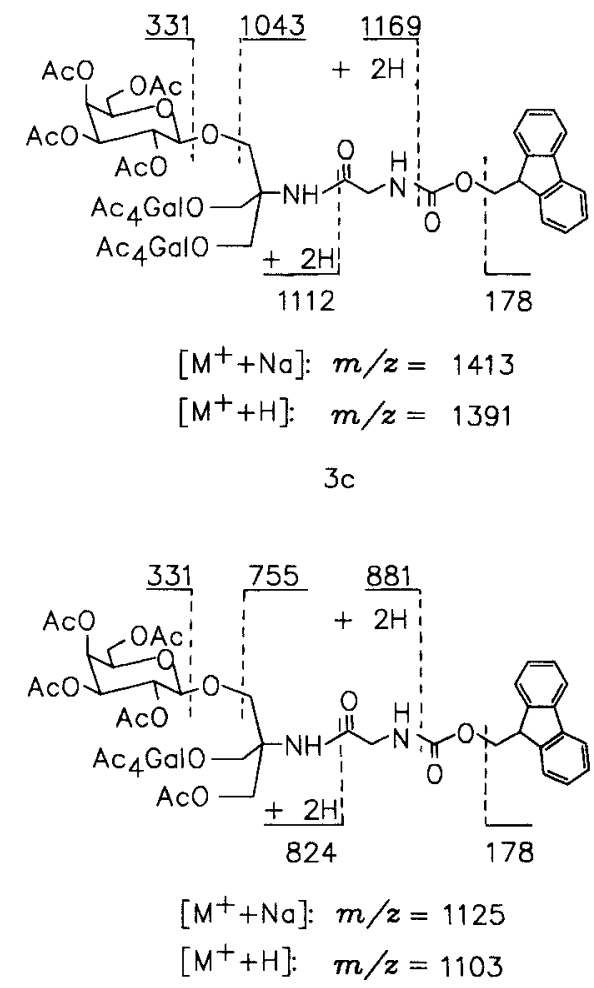

$3 d$

Abb. 1. FAB-MS-Zerfallsmuster von 3e und 3d

Die zur Synthese der peracetylierten Galactose-Cluster vom Typ 6 angewendeten Synthesestrategien sind nachfolgend jeweils anhand von Abb. 2 erläutert. Zur Herstellung von $6 \mathrm{a}$ wird der Block $(\mathrm{A}+\mathrm{B}) \cong \mathbf{3 e}$ mit dem Block $(\mathrm{C}+$ E) $\hat{=}$ 5a verknüpft. Dazu stellt man zunächst das aus der Literatur ${ }^{16)}$ bekannte Hydrogensuccinat 5a aus Bernsteinsäureanhydrid $(\hat{=}$ ) und Cholesterin $(\hat{=}$ E) her. Die Aktivierung von 5a erfolgt mit dem von Steglich et al. ${ }^{17)}$ in die Peptid-Synthese eingeführten cyclischen Carbonat 4 (siehe Schema 1). Die Kopplung von 3e mit 5b (vgl. Lit. ${ }^{18)}$ ) liefert 6a in 87 proz. Ausbeute.

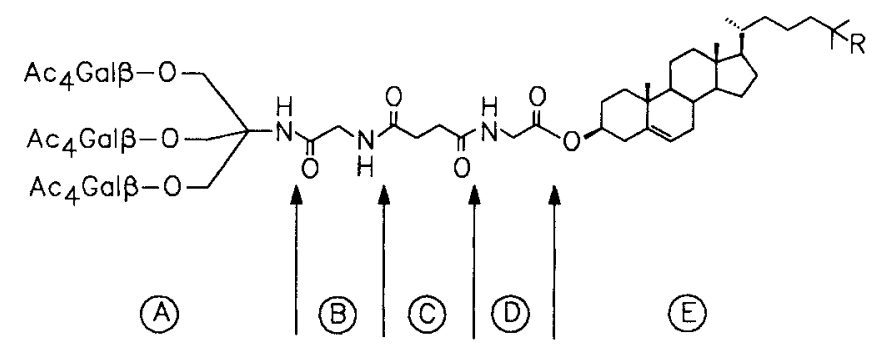

Abb. 2. Synthesebausteine zur Darstellung von 6 


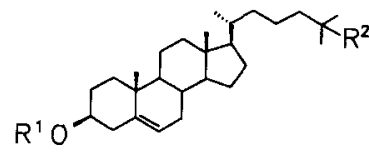

5

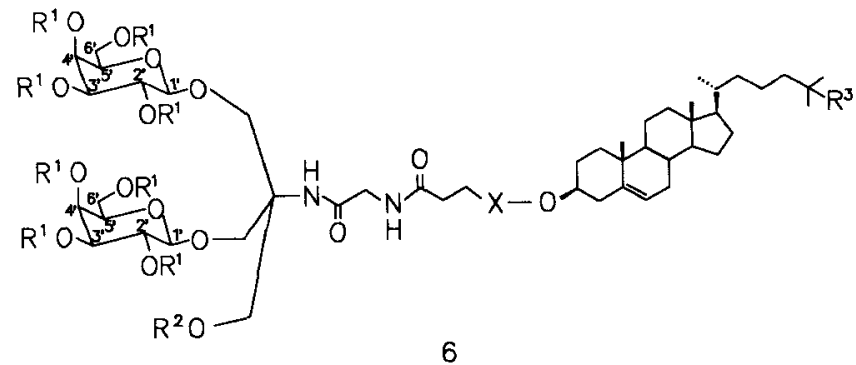

Sowohl die ${ }^{1} \mathrm{H}$ - als auch die ${ }^{13} \mathrm{C}-\mathrm{NMR}$-Spektren von $6 \mathrm{a}$ zeigen die erwarteten Signale. Die 6'-H-Protonen der Tetraacetylgalactosid-Reste werden als Multiplett zusammen mit einem der diastereotopen Methylen-Protonen der Oxymethylen-Gruppe des TRIS-Rests beobachtet. Im FABMassenspektrum erscheint der $\left[\mathrm{M}+\mathrm{H}^{+}\right]$-Peak bei $m / z=$ 1637. Im übrigen steht das Fragmentmuster in Einklang mit der Struktur 6a. Der bei $m / z=331$ beobachtete Basispeak resultiert aus der Fragmentierung der glycosidischen Bindung.

Auch bei der Synthese des im Vergleich zu 6a um eine Glycin-Einheit verlängerten 6d wird der konvergente Weg beschritten. Zunächst baut man durch Umsetzung von 3e mit Bernsteinsäureanhydrid den Block $(\mathrm{A}+\mathrm{B}+\mathrm{C}) \bumpeq \mathbf{3 f}$ in 84 proz. Ausbeute auf. Die Ester-Bindung des Blocks (D $+\mathrm{E}) \hat{=} \mathbf{5 c}$ wird bei der Reaktion von Cholesterin mit BocGly-TDO geknüpft. Nach Schutzgruppen-Abspaltung von 5c mittels Trifluoressigsäure erfolgt die Kopplung von 3f mit 5d in Gegenwart von EEDQ mit 94 proz. Ausbeute.

Der leichte Zugang zu 3f und die Möglichkeit, EsterBindungen über TDO-aktivierte Carbonsäuren herzustellen $^{18)}$, legt einen linearen Syntheseweg $(A+B+C+$ E) für den acetylierten Galactose-Cluster $\mathbf{6 f}$ nahe. Der aus 3f entstandene TDO-Ester 3g wird nicht isoliert. In einer Eintopfreaktion setzt man mit 25-Hydroxycholesterin (5e) um und isoliert anschließend $6 \mathrm{f}$ in 53 proz. Ausbeute. Die selektive Veresterung an C-3 von $5 \mathrm{e}$ folgt aus den ${ }^{13} \mathrm{C}-\mathrm{NMR}$ Spektren von 25-Hydroxycholesterin (5e) und $\mathbf{6 f}$ aus der Tieffeldverschiebung der Resonanz von C-3 in $6 f(\delta=$ 74.23) gegenüber der in $5 \mathbf{e}(\delta=70.20)$.
Die Anwesenheit der Acetyl-Gruppen in den GalactoseResten schließt bei der Umsetzung von aktivierten AcylKomponenten Konkurrenzreaktionen mit freien OH-Gruppen an den Zucker-Resten aus. Außerdem zeigen die peracetylierten Derivate günstige Löslichkeitseigenschaften, was sowohl die Reaktionsführung, als auch die Aufarbeitung und die NMR-spektroskopische Charakterisierung der Verbindungen erleichtert. Diesen Vorteilen steht die Notwendigkeit der Abspaltung der Acetyl-Gruppen in Gegenwart anderer Ester-Funktionen im letzten Schritt der Synthese gegenüber. Die üblichen Methoden, z.B. Einwirkung von Methanolat, Kaliumcarbonat, Kaliumcyanid oder Hydrazinhydrat in Methanol, führen zur raschen Abspaltung des Steroids. Eine selektive Hydrazinolyse der Acetyl-Gruppen gelingt jedoch mit akzeptabler Ausbeute $(33-36 \%$ ) bei tiefen Temperaturen mit $0.1-0.6$ proz. wasserfreiem Hydrazin in absol. Methanol (vgl. Lit. $\left.{ }^{19}\right)$. So erhält man aus 6a in 33 proz. Ausbeute den Galactose-Cluster $(\mathrm{Gal})_{3}$-TRIS-GlySUCC-Chol (6b) und aus 6d bzw. 6f die entsprechenden Verbindungen (Gal) ${ }_{3}$-TRIS-Gly-SUCC-Gly-Chol (6e) bzw. $(\mathrm{Gal})_{3}$-TRIS-Gly-SUCC-(25-OH-Chol) (6g) in 36 proz. Ausbeute. Bei der Hydrazinolyse entstehen außerdem partiell acetyliete Derivate, wie im Falle der Reaktion von 6a durch Isolierung des Monoacetats (AcGal) (Gal) SUCC-Chol (6c) in 25 proz. Ausbeute gezeigt wird. Bei erneuter Durchführung der Hydrazinolyse entsteht $\mathbf{6 b}$ aus $\mathbf{6 c}$ in 45 proz. Ausbeute. Bemerkenswert erscheint, daß die Galactose-Cluster-haltigen Verbindungen als Hydrate anfallen.

Die Resultate dieser Arbeit zeigen, daß komplexe Galactose-Cluster des Typs 6 sowohl konvergent als auch linear aufgebaut werden können. Die Wahl der Synthesestrategie wird im Einzelfall von ökonomischen Überlegungen unter Berücksichtigung der Verfügbarkeit der Wirkstoff-Komponente oder der Einführung einer Isotopenmarkierung bestimmt werden. Über Ergebnisse von derzeit laufenden zellbiologischen Stoffwechseluntersuchungen mit den Galactose-Clustern 6b, 6e und $6 \mathbf{f}$ werden wir an anderer Stelle berichten.

Wir danken der Fa. Merz + Co. GmbH \& Co., Frankfurt, für die großzügige Unterstützung dieser Arbeit und dem Fonds der Chemischen Industrie für eine Forschungsbeihilfe.

\section{Experimenteller Teil}

Eindampfoperationen im Rotationsverdampfer mit Wasserstrahlpumpenvakuum; Badtemp. ca. $30^{\circ} \mathrm{C}$. - DC: Aluminium-Folien mit Kieselgel Si-60 HF 254 (Merck); Laufmittel: DC-A: Chloroform/MeOH (9:1); DC-B: Chloroform/Aceton (8:2); DC-C: Chloroform/MeOH/Wasser (54:40:6); DC-D: Benzol/EtOH (8:2); DCE: Cyclohexan/Ethylacetat (8:2); DC-F: Chloroform/MeOH/Wasser $/ 25 \mathrm{proz}$. Ammoniak (75:25:3:1); DC-G: Toluol/EtOH (9:1); DC-H: Chloroform/Aceton (3:1); DC-I: Ethylacetat/Cyclohexan (2:1); Detektion mittels Fluoreszenzlöschung bei $254 \mathrm{~nm}$ oder nach Anfärben mit 30 proz. methanolischer Schwefelsäure bzw. alkoholischer Ninhydrin-Lösung und nachfolgendem Erhitzen. - SC: Kieselgel Si-60 (Merck), Korngröße 40-60 $\mu \mathrm{m}$. - Schmelzpunkte: Büchi-SMP-20-Apparat. - NMR: Bruker WM 200 oder WM 400; interner Standard bei ${ }^{1} \mathrm{H}-\mathrm{NMR}$ Messungen: Tetramethylsilan; bei ${ }^{13}$ C-NMR Messungen: Lösungsmittel-Signale. Soweit erforderlich, 
wurden die Zuordnungen der Signale in ${ }^{1} \mathrm{H}-\mathrm{NMR}-\mathrm{Spektren}$ mit Hilfe von 2D-COSY- und in ${ }^{13} \mathrm{C}-\mathrm{NMR}-\mathrm{Spektren}$ mit DEPT-90und DEPT-135-Experimenten abgeleitet; exemplarisch ist die vollständige Zuordnung für das ${ }^{13} \mathrm{C}-\mathrm{NMR}$-Spektrum von 6 a (Abschnitt 8) angegeben. - FAB-MS: ZAB-HF-Massenspektrometer umgekehrter Geometrie (VG Analytical, Manchester); methanolische Lösungen der Proben wurden mit Thioglycerin (2,3-Dihydroxy-1-propanthiol) vermischt und mit Xenon-Atomen der kinetischen Energie äquivalent $9 \mathrm{keV}$ bombardiert ${ }^{20)}$; Positiv-Ionen-Spektren wurden im massenkontrollierten Scan von 300-500 s Dauer registriert; die Auswertung der Spektren erfolgte durch Auszählen der Spektrallinien. Bei den Massenangaben handelt es sich daher um Nominalmassen. - TMS-Triflat und TRIS wurden von Merck, Pentaacetylgalactose von Sigma bezogen. Acetobromgalactose ${ }^{9)}$, BocGly-O-TDO ${ }^{21)}$, Cholesterylhydrogensuccinat ${ }^{9}$, EEDQ ${ }^{22)}$, FmocGlycin ${ }^{23)}$, und $4^{17)}$ wurden nach Literaturangaben hergestellt. 25 Hydroxycholesterin wurde aus Stigmasterin synthetisiert ${ }^{24)}$.

1) $N^{x}$-[(9-Fluorenyl)methoxycarbonyl]-N-[2-hydroxy-1,1-bis(hydroxymethyl)ethylgglycinamid (TRIS-Gly-Fmoc, 2 b): Eine Lösung von $2.24 \mathrm{~g}(18.5 \mathrm{mmol})$ TRIS, $5.02 \mathrm{~g}(16.9 \mathrm{mmol}) \mathbf{1 ~ b}$ und $5.1 \mathrm{~g}$ (20.8 mmol) EEDQ in $165 \mathrm{ml}$ absol. EtOH wird unter Argon $5 \mathrm{~h}$ unter Rückfluß erhitzt. Danach wird eingeengt und der Rückstand mit $150 \mathrm{ml}$ Diethylether versetzt. Man saugt ab und wäscht die Kristalle mehrmals mit absol. Ether. Das Rohprodukt wird in $\mathrm{MeOH}$ aufgenommen und bis zur Neutralreaktion mit Dowex $50 \mathrm{~W}$ $\mathrm{X}-200\left(\mathrm{H}^{\oplus}\right.$-Form) versetzt. Nach dem Filtrieren wird die Lösung zur Trockene eingeengt. 2 b kristallisiert nach Vermischen des Rückstandes mit Diethylether aus. Man läBt noch $12 \mathrm{~h}$ bei $-20^{\circ} \mathrm{C}$ stehen, filtriert durch eine Glasfritte und trocknet über Kaliumhydroxid und Paraffin bei 0.2 Torr. - Ausb. 3.72 g (55\%), Schmp. $85-87^{\circ} \mathrm{C}$ - DC-A: $R_{\mathrm{f}}=0.31 .-{ }^{1} \mathrm{H}-\mathrm{NMR}\left(200 \mathrm{MHz},\left[\mathrm{D}_{6}\right]-\right.$ DMSO): $\delta=7.90(\mathrm{~d}, J=7.2 \mathrm{~Hz}, 2$ aromat. $\mathrm{H}), 7.71(\mathrm{~d}, J=7.2$ $\mathrm{Hz}, 2$ aromat. $\mathrm{H}), 7.56(\mathrm{t}, J=5.9 \mathrm{~Hz}, 1 \mathrm{H}, \mathrm{NH}), 7.47-7.28(\mathrm{~m}, 4$ aromat. $\mathrm{H}$ ), 7.19 (br. s, $1 \mathrm{H}, \mathrm{NH}), 4.72$ (t, $J=5.8 \mathrm{~Hz}, 3 \mathrm{H}, \mathrm{OH}$ ), $4.33-4.16\left(\mathrm{~m}, 3 \mathrm{H}, \mathrm{CH}_{2} \mathrm{CH}=\right), 3.66(\mathrm{~d}, J=5.9 \mathrm{~Hz}, 2 \mathrm{H}$, $\mathrm{COCH}_{2} \mathrm{NH}$ ), 3.54 (d, $J=5.8 \mathrm{~Hz}, 6 \mathrm{H}$, $\left.\equiv \mathrm{CCH}_{2} \mathrm{O}\right) .-{ }^{13} \mathrm{C}-\mathrm{NMR}$ (50.3 MHz, [D $\left.\left.\mathrm{D}_{6}\right] \mathrm{DMSO}\right): \delta=170.00$ (NCOC), 156.48 (NCOO), $143.85,140.75,127.67,127.12,125.28,120.13$ (aromat. C), 63.32 $(\equiv \mathrm{C}), 62.16\left(\equiv \mathrm{CCH}_{2} \mathrm{O}\right), 60.34\left(\mathrm{OCH}_{2} \mathrm{CH}=\right), 46.62\left(\mathrm{CH}_{2} \mathrm{CH}=\right)$, $43.90\left(\mathrm{NCH}_{2} \mathrm{CO}\right)$.

$\mathrm{C}_{21} \mathrm{H}_{24} \mathrm{~N}_{2} \mathrm{O}_{6} \cdot 0.5 \mathrm{H}_{2} \mathrm{O}(409.4) \quad$ Ber. C $61.60 \mathrm{H} 6.15 \mathrm{~N} 6.84$ Gef. C 61.57 H 6.29 N 6.33

2) $N^{\alpha}-[$ (9-Fluorenyl)methoxycarbonyl]-N-[tris(2,3,4,6-tetra-Oacet $y l-\beta$-D-galactopyranosylox ymethyl) methyl glycinamid $\left[\left(\mathrm{Ac}_{4} \mathrm{Gal}_{3}\right)_{3}\right.$-TRIS-Gly-Fmoc, 3c]: $\mathrm{Zu}$ einer unter Argon kräftig gerührten Suspension von $2.8 \mathrm{~g}(6.9 \mathrm{mmol}) \mathbf{2}$ b sowie $2.9 \mathrm{~g}(10.6 \mathrm{mmol})$ $\mathrm{Hg}(\mathrm{CN})_{2}$ und $3.8 \mathrm{~g}$ (10.6 mmol) $\mathrm{HgBr}_{2}$ in $80 \mathrm{ml}$ Acetonitril wird innerhalb von 60 min bei Raumtemp. eine Lösung von $8.75 \mathrm{~g} \mathrm{(21.3}$ mmol) 2,3,4,6-Tetra- $O$-acetyl-1-brom-1-desoxy- $\alpha$-D-galactose in $80 \mathrm{ml}$ Acetonitril getropft. Nach weiteren $30 \mathrm{~min}$ fügt man zu der klaren Lösung nochmals $0.9 \mathrm{~g}(3.5 \mathrm{mmol}) \mathrm{Hg}(\mathrm{CN})_{2}, 1.3 \mathrm{~g}(3.5 \mathrm{mmol})$ $\mathrm{HgBr}_{2}$ und $2.9 \mathrm{~g}(7.1 \mathrm{mmol})$ Acetobromgalactose. Anschließend läßt man $24 \mathrm{~h}$ bei $20^{\circ} \mathrm{C}$ rühren. Dann wird zur Trockne eingedampft und der Rückstand in $250 \mathrm{~m} 1$ Chloroform aufgenommen. Nach dem Waschen mit 12 proz. Kaliumbromid-Lösung $(5 \times 100 \mathrm{ml})$ und Wasser $(5 \times 100 \mathrm{ml})$ wird mit Magnesiumsulfat getrocknet und eingedampft. Das sirupöse Rohprodukt (13.9 g) liefert nach SC [Etylacetat/Cyclohexan (2:1)] und Trocknen über $\mathrm{P}_{4} \mathrm{O}_{10} 3 \mathrm{c}$ als farbloses Pulver. - Ausb. $3.75 \mathrm{~g}$ (38\%), Schmp. $126-129^{\circ} \mathrm{C}$. DC-B: $R_{\mathrm{f}}=0.36$. DC-D: $R_{\mathrm{f}}=0.65$. DC-I: $R_{\mathrm{f}}=0.21$. $[\alpha]_{\mathrm{D}}^{20}=-9.3\left(c=1\right.$ in $\left.\mathrm{CHCl}_{3}\right) \cdot-{ }^{1} \mathrm{H}-\mathrm{NMR}\left(400 \mathrm{MHz}, \mathrm{CDCl}_{3}\right)$ : $\delta=7.68(\mathrm{~d}, J=7.5 \mathrm{~Hz}, 2$ aromat. $\mathrm{H}), 7.55\left(\mathrm{ddd}, J_{o}=7.5 \mathrm{~Hz}\right.$, $J_{m}=2 \mathrm{~Hz}, J_{p}=0.8 \mathrm{~Hz}, 2$ aromat. $\left.\mathrm{H}\right), 7.32\left(\mathrm{dt}, J_{o}=7.0 \mathrm{~Hz}, J_{p}=\right.$
$0.8 \mathrm{~Hz}, 2$ aromat. $\mathrm{H}), 7.24(\mathrm{~m}, 2$ aromat. $\mathrm{H}), 6.19(\mathrm{~s}, 1 \mathrm{H}, \mathrm{NH}), 5.71$ (br. t, $J=6.0 \mathrm{~Hz}, 1 \mathrm{H}, \mathrm{NH}), 5.31\left(\mathrm{dd}, J_{3,4^{\prime}}=2.8 \mathrm{~Hz}, J_{4^{\prime}, 5^{\prime}}=0.7\right.$ $\left.\mathrm{Hz}, 3 \mathrm{H}, 4^{\prime}-\mathrm{H}\right), 5.07$ (dd, $\left.J_{2^{\prime}, 3^{\prime}}=10.5 \mathrm{~Hz}, J_{1^{\prime}, 2^{\prime}}=7.7 \mathrm{~Hz}, 3 \mathrm{H}, 2^{\prime}-\mathrm{H}\right)$, $4.96\left(\mathrm{dd}, J_{2} \cdot 3^{\prime}=10.5 \mathrm{~Hz}, J_{3^{\prime}, 4^{\prime}}=3.3 \mathrm{~Hz}, 3 \mathrm{H}, 3^{\prime}-\mathrm{H}\right), 4.36\left(\mathrm{~d}, J_{1^{\prime}, 2^{\prime}}=\right.$ $\left.7.7 \mathrm{~Hz}, 3 \mathrm{H}, 1^{\prime}-\mathrm{H}\right), 4.32\left(\mathrm{dd}, J_{\text {gem. }}=7.5 \mathrm{~Hz}, J_{\text {vic. }}=4.3 \mathrm{~Hz}, 2 \mathrm{H}\right.$, $\left.\mathrm{CH}_{2} \mathrm{CH}=\right), 4.16\left(\mathrm{t}, J_{\text {vic. }}=7.5 \mathrm{~Hz}, 1 \mathrm{H}, \mathrm{CH}_{2} \mathrm{CH}=\right), 4.12-4.01(\mathrm{~m}$, $9 \mathrm{H}, 6^{\prime}-\mathrm{H}$ und $\left.\equiv \mathrm{CCH}_{\mathrm{A}} \mathrm{HO}\right), 3.86\left(\mathrm{t}, J_{5^{\prime}, 6^{\prime}}=7.0 \mathrm{~Hz}, 3 \mathrm{H}, 5^{\prime}-\mathrm{H}\right), 3.76$ $\left(\mathrm{dd}, J=10.8 \mathrm{~Hz}, J=6.0 \mathrm{~Hz}, 2 \mathrm{H}, \mathrm{NHCH}_{2}\right), 3.72(\mathrm{~d}, J=10.5 \mathrm{~Hz}$, $\left.3 \mathrm{H}, \equiv \mathrm{CCH}_{\mathrm{B}} \mathrm{HO}\right), 2.11,2.03,2.02,1.94\left(4 \mathrm{~s}, 36 \mathrm{H}, \mathrm{CH}_{3} \mathrm{CO}\right) .-{ }^{13} \mathrm{C}-$ NMR $\left(100 \mathrm{MHz}_{1} \mathrm{CDCl}_{3}\right): \delta=170.25,170.02,169.81,169.36,168.77$ $\left(\mathrm{CH}_{3} \mathrm{C}=\mathrm{O}\right.$ und $\left.\mathrm{NCOCH}_{2}\right), 156.29\left(\mathrm{NCO}_{2}\right), 143.69,141.07,127.56$, $126.95,124.98,119.84$ (aromat. C), $101.16\left(\mathrm{C}^{\prime} \mathbf{1}^{\prime}\right), 70.65,70.35$ (C-3' und $\left.\mathrm{C}-5^{\prime}\right), 68.87\left(\mathrm{C}-2^{\prime}\right), 68.06\left(\mathrm{OCH}_{2}\right), 66.82\left(\mathrm{C}-4^{\prime}\right), 61.02\left(\mathrm{C}-6^{\prime}\right)$ und $\left.\mathrm{COCH}_{2} \mathrm{CH}=\right), 59.04(\equiv \mathrm{C}), 46.92\left(\mathrm{CH}_{2} \mathrm{CH}=\right), 44.09\left(\mathrm{NHCH}_{2} \mathrm{CO}\right)$, 20.63, 20.50, 20.44, $20.38\left(\mathrm{CH}_{3} \mathrm{CO}\right) .-\mathrm{FAB}-\mathrm{MS}: m / z(\%)=1413$ (9.5) $\left[\mathrm{M}+\mathrm{Na}^{+}\right], 1391$ (1) $\left[\mathrm{M}+\mathrm{H}^{+}\right], 1371$ (2), 1353 (1), $1311(0.3)$, 1191 (1), 1125 (2), 1112 (1), 1083 (1), 1061 (1), 1043 (5), 1001 (1), 865 (0.3), 771 (1), 755 (1), 713 (1), 331 (54), 289 (8), 229 (11), 178 (87), 169 (100).

\section{$\mathrm{C}_{63} \mathrm{H}_{78} \mathrm{~N}_{2} \mathrm{O}_{33} \cdot 2.5 \mathrm{H}_{2} \mathrm{O}(1427.3) \quad$ Ber. C $52.68 \mathrm{H} 5.82 \mathrm{~N} 1.95$ Gef. C 52.70 H 5.55 N 1.96}

3) $\mathrm{N}$-[ Tris (2,3,4,6-tetra-O-acetyl- $\beta$-D-galactopyranosyloxymethyl) methyl $]$ glycinamid $\left[\left(\mathrm{Ac}_{4} \mathrm{Gal}\right)_{3}\right.$-TRIS-Gly, 3e]: Man rührt $2.1 \mathrm{~g}$ $(1.5 \mathrm{mmol}) 3 \mathrm{c}$ in $50 \mathrm{ml}$ einer 10 proz. Lösung von Piperidin in absol. Dichlormethan unter Argon $90 \mathrm{~min}$ bei $20^{\circ} \mathrm{C}$. Anschließend wird mit Dichlormethan auf $100 \mathrm{ml}$ verdünnt und mit $0.75 \mathrm{~N} \mathrm{HCl}(3 \times$ $50 \mathrm{ml}$ ) gewaschen. Die mit $\mathrm{MgSO}_{4}$ getrocknete organische Phase wird bis auf etwa $0.5 \mathrm{ml}$ eingeengt und anschließend mit $10 \mathrm{ml}$ Diethylether vermischt. Man läßt ca. $12 \mathrm{~h}$ bei $-20^{\circ} \mathrm{C}$ stehen und saugt ab. Das Rohprodukt wird ohne weitere Reinigung für die anschließende Reaktion verwendet. - Ausb. $1.6 \mathrm{~g}(93 \%)$, Schmp. $124-126^{\circ} \mathrm{C}$ (Zers.). - DC-A: $R_{\mathrm{f}}=0.44$. - Zur Analyse wird eine Probe der SC [Chloroform/MeOH (9:1)] unterworfen und aus Diethylether/Pentan kristallisiert. $-[\alpha]_{\mathrm{D}}^{20}=-14.1(c=1$ in $\mathrm{MeOH}) .-{ }^{1} \mathrm{H}-\mathrm{NMR}\left(200 \mathrm{MHz}, \mathrm{CDCl}_{3}\right): \delta=5.39$ (d, $J_{3^{\prime}, 4^{\prime}}=3.8$ $\left.\mathrm{Hz}, 3 \mathrm{H}, 4^{\prime}-\mathrm{H}\right), 5.15\left(\mathrm{dd}, J_{2^{\prime}, 3^{\prime}}=10.4 \mathrm{~Hz}, J_{2^{\prime}, 1^{\prime}}=7.7 \mathrm{~Hz}, 3 \mathrm{H}, 2^{\prime}-\mathrm{H}\right)$, $5.01\left(\mathrm{dd}, J_{2^{\prime}, 3^{\prime}}=10.5 \mathrm{~Hz}, J_{3^{\prime}, 4^{\prime}}=3.8 \mathrm{~Hz}, 3 \mathrm{H}, 3^{\prime}-\mathrm{H}\right), 4.44\left(\mathrm{~d}, J_{1^{\prime} 2^{\prime}}=\right.$ $\left.7.8 \mathrm{~Hz}, 3 \mathrm{H}, 1^{\prime}-\mathrm{H}\right), 4.41\left(\mathrm{~m}, 9 \mathrm{H}, 6^{\prime}-\mathrm{H}\right.$ und $\left.\equiv \mathrm{CCH}_{\mathrm{A}} \mathrm{HO}\right), 3.92(\mathrm{t}, J=$ $\left.7 \mathrm{~Hz}, 3 \mathrm{H}, 5^{\prime}-\mathrm{H}\right), 3.83\left(\mathrm{~d}, J=10.4 \mathrm{~Hz}, 3 \mathrm{H}\right.$, $\left.\equiv \mathrm{CCH}_{\mathrm{B}} \mathrm{HO}\right), 3.26(\mathrm{~s}$, $\left.2 \mathrm{H}, \mathrm{COCH}_{2} \mathrm{NH}_{2}\right), 2.17,2.07,2.06,1.99\left(4 \mathrm{~s}, 36 \mathrm{H}, \mathrm{CH}_{3} \mathrm{CO}\right)$. - FABMS: $m / z(\%)=1191(1)\left[\mathrm{M}+\mathrm{Na}^{+}\right], 1169[11]\left[\mathrm{M}+\mathrm{H}^{+}\right], 1127$ (3), 1112 (2), 839 (2), 821 (7), 779 (2), 491 (1), 331 (88), 289 (12), 265 (18), $229(14), 169(100)$.

$$
\begin{array}{lll}
\mathrm{C}_{48} \mathrm{H}_{68} \mathrm{~N}_{2} \mathrm{O}_{31}(1169.1) & \text { Ber. C } 49.32 \text { H } 5.86 \text { N } 2.40 \\
& \text { Gef. C } 49.41 \text { H } 5.87 \text { N } 2.22
\end{array}
$$

4) $N^{\alpha}$-Succinyl-N-[tris (2,3,4,6-tetra-O-acetyl- $\beta$-D-galactopyranosyloxymethyl)methyl glycinamid $\left[\left(\mathrm{Ac}_{4} \mathrm{Gal}\right)_{3}\right.$-TRIS-Gly-SUCC, 3]]: $2.0 \mathrm{~g}(1.7 \mathrm{mmol}) 3 \mathrm{e}$ und $1.7 \mathrm{~g}(17 \mathrm{mmol})$ Bernsteinsäureanhydrid werden in $50 \mathrm{ml}$ Dichlormethan suspendiert und mit $0.29 \mathrm{ml} \mathrm{N}$ Ethyldiisopropylamin $(1.7 \mathrm{mmol})$ versetzt. Anschließend rührt man $24 \mathrm{~h}$ bei $20^{\circ} \mathrm{C}$. Die Suspension wird mit $150 \mathrm{ml}$ Dichlormethan verdünnt und mit $1 \mathrm{~N} \mathrm{HCl}(3 \times 100 \mathrm{ml})$ gewaschen. Die mit Magnesiumsulfat getrocknete organische Phase wird zur Trockne eingedampft und der sirupöse Rückstand mit $10 \mathrm{ml}$ Pentan verrührt. Nach Absaugen des Niederschlages wird mittels SC [Chloroform/ $\mathrm{MeOH}(9: 1)]$ gereinigt und über $\mathrm{P}_{4} \mathrm{O}_{10}$ getrocknet. Man erhält 3f als farblose Kristalle. - Ausb. $1.9 \mathrm{~g}(84 \%)$, Schmp. $107-110^{\circ} \mathrm{C}$. DC-F: $R_{\mathrm{f}}=0.36 .-[\alpha]_{\mathrm{D}}^{20}=-18.3\left(c=1\right.$ in $\left.\mathrm{CHCl}_{3}\right) .-{ }^{1} \mathrm{H}-\mathrm{NMR}$ $\left(200 \mathrm{MHz}, \mathrm{CDCl}_{3}\right): \delta=6.97$ (br. s, $\left.1 \mathrm{H}, \mathrm{NH}\right), 6.38$ (br. s, $1 \mathrm{H}, \mathrm{NH}$ ), $5.31\left(\mathrm{~d}, J=2.5 \mathrm{~Hz}, 3 \mathrm{H}, 4^{\prime}-\mathrm{H}\right), 5.12-4.90\left(\mathrm{~m}, 6 \mathrm{H}, 2^{\prime}-\right.$ und $\left.3^{\prime}-\mathrm{H}\right)$, $4.40\left(\mathrm{~d}, J=6.2 \mathrm{~Hz}, 3 \mathrm{H}, 1^{\prime}-\mathrm{H}\right), 4.21-3.58\left(\mathrm{~m}, 17 \mathrm{H}, \equiv \mathrm{CCH}_{2} \mathrm{O}, 5^{\prime}-\right.$ und $6^{\prime}-\mathrm{H}$ und $\left.\mathrm{NHCH}_{2} \mathrm{CO}\right), 2.67-2.40\left(\mathrm{~m}, 4 \mathrm{H}, \mathrm{COCH}_{2} \mathrm{CH}_{2} \mathrm{CO}\right)$, $2.11,2.01,1.91\left(3 \mathrm{~s}, 36 \mathrm{H}, \mathrm{H}_{3} \mathrm{CCO}\right) .-{ }^{13} \mathrm{C}-\mathrm{NMR}\left(50.3 \mathrm{MHz}, \mathrm{CDCl}_{3}\right)$ : 
$\delta=172.83,170.51,170.19,170.02,169.63,169.39(\mathrm{C}=\mathrm{O}), 101.29$, $70.67,70.47,69.03,67.89,66.94,61.06,59.46,42.88,30.71,30.25$, $20.75,20.64,20.58,20.51$.

$$
\begin{array}{ll}
\mathrm{C}_{52} \mathrm{H}_{72} \mathrm{~N}_{2} \mathrm{O}_{34} \cdot 3 \mathrm{H}_{2} \mathrm{O}(1323.2) & \text { Ber. C } 47.20 \mathrm{H} 5.94 \mathrm{~N} 2.12 \\
& \text { Gef. C } 47.30 \mathrm{H} 5.51 \mathrm{~N} 1.61
\end{array}
$$

5) (5-Cholesten-3 $\beta-y l)$ (TDO) succinat (5b): Zu einer Lösung von $1.39 \mathrm{~g}(4.26 \mathrm{mmol}) \mathbf{4}$ und $2.08 \mathrm{~g}(4.26 \mathrm{mmol}) \mathbf{5 a}$ in $25 \mathrm{ml}$ Dichlormethan fügt man bei $20^{\circ} \mathrm{C} 0.312 \mathrm{ml}(4 \mathrm{mmol})$ Pyridin. Nach $24 \mathrm{~h}$ wird mit $75 \mathrm{ml}$ Dichlormethan verdünnt und wie folgt extrahiert (vgl. Lit. $\left.{ }^{17}\right)$ : 20 proz. wäßrige Citronensäure-Lösung $(3 \times 50 \mathrm{ml})$, ges. Natriumhydrogencarbonat-Lösung $(10 \times 50 \mathrm{ml})$, 20proz. wäßrige Citronensäure-Lösung $(1 \times 50 \mathrm{ml})$ und Wasser $(3 \times 50 \mathrm{ml})$. Die mit Magnesiumsulfat getrocknete organische Phase wird eingedampft. - Ausb. $2.1 \mathrm{~g} \mathrm{(64 \% ),} \mathrm{Schmp.} 136-138^{\circ} \mathrm{C}$ (Zers.). DC-E: $R_{\mathrm{f}}=0.26 .-[\alpha]_{\mathrm{D}}^{20}=-27.5\left(c=1\right.$ in $\left.\mathrm{CHCl}_{3}\right) .-{ }^{1} \mathrm{H}-\mathrm{NMR}$ $\left(200 \mathrm{MHz}, \mathrm{CDCl}_{3}\right): \delta=7.97(\mathrm{~m}, 2$ aromat. $\mathrm{H}), 7.55(\mathrm{~m}, 3$ aromat. $\mathrm{H}), 7.44(\mathrm{~m}, 3$ aromat. $\mathrm{H}), 7.33(\mathrm{~m}, 2$ aromat. $\mathrm{H}), 5.36(\mathrm{~m}, 1 \mathrm{H}, 6-\mathrm{H}$ von Chol), 5.21 (s, $1 \mathrm{H}, \mathrm{H}$ von Thiophen), 4.63 (br. $\mathrm{m}, 1 \mathrm{H}, 3-\mathrm{H}$ von Chol), $2.98\left(\mathrm{t}, J=6.2 \mathrm{~Hz}, 2 \mathrm{H}, \mathrm{COCH}_{2} \mathrm{CH}_{2} \mathrm{CO}\right), 2.70(\mathrm{t}, J=6.2$ $\left.\mathrm{Hz}, 2 \mathrm{H}, \mathrm{COCH}_{2} \mathrm{CH}_{2} \mathrm{CO}\right), 2.31$ (d, $J=7.5 \mathrm{~Hz}, 2 \mathrm{H}, 4-\mathrm{H}$ von $\mathrm{Chol}$ ), $2.1-0.7(\mathrm{~m}, 41 \mathrm{H}) .-{ }^{13} \mathrm{C}-\mathrm{NMR}\left(50.3 \mathrm{MHz}, \mathrm{CDCl}_{3}\right): \delta=184.54$, 170.79, $168.67(\mathrm{C}=\mathrm{O}), 148.23,145.35$ (aromat. C), 139.39 (C-5 von Chol), 132.71, 130.84, 129.91, 129.58, 129.43, 129.25 (aromat. C), $127.13,123.76$ ( $\mathrm{C}=\mathrm{C}$ von Thiophen), 122.78, 74.77, 56.65, 56.10, $49.95,43.02,42.27,39.69,39.5,37.96,36.89,36.52,36.17,35.78,31.87$, $31.79,28.98,28.79,28.23,27.99,27.63,24.27,24.83,22.85,22.59$, $21.00,19.28,18.72,11.86$

$$
\begin{array}{rr}
\mathrm{C}_{47} \mathrm{H}_{60} \mathrm{O}_{7} \mathrm{~S}(796.1) & \text { Ber. C } 73.40 \text { H } 7.86 \\
& \text { Gef. C } 72.85 \text { H } 8.06
\end{array}
$$

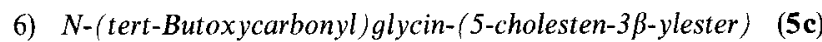
(vgl. Lit. $\left.{ }^{25}\right): 1.03 \mathrm{~g}(2.2 \mathrm{mmol})$ Boc-Gly-O-TDO und $1.2 \mathrm{~g}(3.1 \mathrm{mmol})$ Cholesterin in $50 \mathrm{ml}$ trockenem Dichlormethan werden mit $0.25 \mathrm{ml}$ (2.24 mmol), $N$-Ethyldiisopropylamin versetzt. Man rührt $7 \mathrm{~h}$ bei $20^{\circ} \mathrm{C}$ (Farbumschlag von Rot nach Orange), verdünnt mit $50 \mathrm{ml}$ Dichlormethan und arbeitet wie in Abschnitt 5 beschrieben auf. SC [Cyclohexan/Essigester (1:1)] liefert 5c. - Ausb. $1.7 \mathrm{~g} \mathrm{(70 \% ),}$ Schmp. $112-114^{\circ} \mathrm{C}$. DC-E: $R_{\mathrm{f}}=0.52 .-{ }^{1} \mathrm{H}-\mathrm{NMR}(400 \mathrm{MHz}$, $\left.\mathrm{CD}_{2} \mathrm{Cl}_{2}\right): \delta=5.36(\mathrm{~m}, 1 \mathrm{H}, 6-\mathrm{H}), 5.15(\mathrm{t}, J=3 \mathrm{~Hz}, 1 \mathrm{H}, \mathrm{NH}), 4.6$ $(\mathrm{m}, 1 \mathrm{H}, 3-\mathrm{H}), 3.81\left(\mathrm{~d}, J=3 \mathrm{~Hz}, 2 \mathrm{H}, \mathrm{NHCH}_{2} \mathrm{CO}\right), 2.31(\mathrm{~m}, 2 \mathrm{H}, 4$ $\mathrm{H}), 2.04-0.83(\mathrm{~m}$ mit s bei $\delta=1.41,1.00$ und $0.67,50 \mathrm{H}) .-{ }^{13} \mathrm{C}-$ NMR $\left(50.3 \mathrm{MHz}, \mathrm{CD}_{2} \mathrm{Cl}_{2}\right): \delta=170.13,156.04(\mathrm{C}=\mathrm{O}), 139.92$, $132.15,79.86,75.37,57.14,56.61,50.48,43.02,42.7,40.18,39.92$, $38.42,37.35,36.95,36.1,36.25,32.31,32.25 .28 .63,28.47\left[\left(\mathrm{CH}_{3}\right)_{3} \mathrm{C}\right]$, $28.43,28.09,24.66,24.27,23.01,22.77,21.44,19.52,18.96,12.07$.

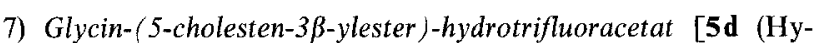
drotrifluoracetat)] (vgl. Lit. $\left.{ }^{25)}\right): 1.0 \mathrm{~g}(1.8 \mathrm{mmol}) 5 \mathrm{c}$ werden mit $2.5 \mathrm{ml}$ Trifluoressigsäure vermischt. Es setzt sofort eine Gasentwicklung ein. Man rührt $1 \mathrm{~h}$ bei $20^{\circ} \mathrm{C}$ und entfernt die Trifluoressigsäure bei 1 Torr. Das zurückbleibende bräunliche Pulver wird ohne weitere Reinigung weiterverarbeitet. - Ausb. $0.98 \mathrm{~g} \mathrm{(96 \% ),}$ Schmp. $185^{\circ} \mathrm{C}$ (Zers). - DC-D: $R_{\mathrm{f}}=0.35$. - ${ }^{1} \mathrm{H}-\mathrm{NMR}(90 \mathrm{MHz}$, $\left.\mathrm{CDCl}_{3},\left[\mathrm{D}_{6}\right] \mathrm{DMSO}\right): \delta=5.13(\mathrm{~m}, 1 \mathrm{H}, 6-\mathrm{H}), 4.4(\mathrm{~m}, 1 \mathrm{H}, 3-\mathrm{H}), 3.5$ $\left(\mathrm{s}, 2 \mathrm{H}, \mathrm{NCH}_{2} \mathrm{CO}\right), 2.5-0.5(43 \mathrm{H})$.

8) $N^{\alpha}-[4-(5-C h o l e s t e n-3 \beta-y l o x y)$ succinyl]- $N$ - $[$ tris (2,3,4,6-tetra-Oacetyl- $\beta$-D-galactopyranosyloxymethyl) methyl Jglycinamid [( $\left(\mathrm{Ac}_{4} \mathrm{Gal}\right)_{3}$-TRIS-Gly-SUCC-Chol, 6a]: $1.35 \mathrm{~g}(1.15 \mathrm{mmol}) 3 \mathrm{e}$ und $0.86 \mathrm{~g}(1.11 \mathrm{mmol}) \mathbf{5 b}$ werden in $100 \mathrm{ml}$ trockenem Dichlormethan gelöst und mit $0.39 \mathrm{ml}(2.24 \mathrm{mmol}) \mathrm{N}$-Ethyldiisopropylamin versetzt. Die Farbe der bei $20^{\circ} \mathrm{C}$ gerührten Reaktionsmischung wechselt innerhalb $6 \mathrm{~h}$ von zunächst Tiefrot nach Orangegelb. Man verdünnt mit $100 \mathrm{ml}$ Dichlormethan und extrahiert wie in $\mathrm{Ah}$ - schnitt 5 beschrieben. Die mit $\mathrm{MgSO}_{4}$ getrocknete organische Phase wird eingedampft und der Rückstand in $10 \mathrm{ml}$ Diethylether/Hexan (1:1) aufgenommen. Der nun erhaltene Feststoff wird abfiltriert und der SC [Chloroform/Aceton (4:1)] unterworfen. Nach dem Trocknen über $\mathrm{P}_{4} \mathrm{O}_{10}$ erhält man 6a. - Ausb. $1.61 \mathrm{~g}(87 \%)$, Schmp. $129-132^{\circ} \mathrm{C}$. DC-B: $R_{\mathrm{f}}=0.39 .-\mathrm{DC}-\mathrm{D}: R_{\mathrm{f}}=0.68 .-[\alpha]_{\mathrm{D}}^{20}=$ $-23.1\left(c=1\right.$ in $\left.\mathrm{CHCl}_{3}\right) .-{ }^{1} \mathrm{H}-\mathrm{NMR}\left(200 \mathrm{MHz}, \mathrm{CDCl}_{3}\right): \delta=6.53$ $(\mathrm{t}, J=5.0 \mathrm{~Hz}, 1 \mathrm{H}, \mathrm{NH}), 6.21(\mathrm{~s}, 1 \mathrm{H}, \mathrm{NH}), 5.39\left(\mathrm{dd}, J_{3^{\prime} 4^{\prime}}=3.1 \mathrm{~Hz}\right.$, $\left.J_{4^{\prime}, 5^{\prime}}=1 \mathrm{~Hz}, 3 \mathrm{H}, 4^{\prime}-\mathrm{H}\right), 5.34(\mathrm{~m}, 1 \mathrm{H}, 6-\mathrm{H}$ von Chol), $5.13(\mathrm{dd}$, $\left.J_{2^{\prime}, 3^{\prime}}=10.5 \mathrm{~Hz}, J_{1^{\prime}, 2^{\prime}}=7.5 \mathrm{~Hz}, 3 \mathrm{H}, 2^{\prime}-\mathrm{H}\right), 5.02\left(\mathrm{dd}, J_{2^{\prime}, 3^{\prime}}=10.5\right.$ $\left.\mathrm{Hz}, J_{3^{\prime}, 4^{\prime}}=3.2 \mathrm{~Hz}, 3 \mathrm{H}, 3^{\prime}-\mathrm{H}\right), 4.6(\mathrm{~m}, 1 \mathrm{H}, 3-\mathrm{H}$ von $\mathrm{Chol}), 4.42(\mathrm{~d}$, $J_{1^{\prime}, z^{\prime}}=7.5 \mathrm{~Hz}, 3 \mathrm{H}, 1^{\prime}-\mathrm{H}$ ), $4.28-4.10$ (m mit d bei $\delta=4.18, J=$ $10.4 \mathrm{~Hz}, 9 \mathrm{H}, 6^{\prime}-\mathrm{H}$ und $\left.\equiv \mathrm{CCH}_{\mathrm{A}} \mathrm{HO}\right), 3.94\left(\mathrm{t}, J_{5^{\prime}, 6^{\prime}}=7.1 \mathrm{~Hz}, 3 \mathrm{H}\right.$, $\left.5^{\prime}-\mathrm{H}\right), 3.86\left(\mathrm{~d}, J=5 \mathrm{~Hz}, 2 \mathrm{H}, \mathrm{NHCH}_{2}\right), 3.76(\mathrm{~d}, J=10.4 \mathrm{~Hz}, 3 \mathrm{H}$, $\left.\equiv \mathrm{CC} H_{\mathrm{B}} \mathrm{HO}\right), 2.61\left(\mathrm{~m}, 4 \mathrm{H}, \mathrm{COCH}_{2} \mathrm{CH}_{2} \mathrm{CO}\right), 2.31(\mathrm{~m}, 2 \mathrm{H}, 4-\mathrm{H}$ von Chol), 2.2-0.7 (m mit $\mathrm{s}$ bei $\delta=2.17,2.07,2.06$ und $1.89,77 \mathrm{H}$, $\mathrm{CH}_{3} \mathrm{CO}$ und $\mathrm{H}$ von Chol). $-{ }^{13} \mathrm{C}-\mathrm{NMR}\left(50.3 \mathrm{MHz}, \mathrm{CDCl}_{3}\right)(\mathrm{Zu}-$ ordnung: vgl. Lit. $\left.{ }^{26)}\right): \delta=172.20\left(\mathrm{CH}_{2} \mathrm{NHCOCH}_{2}\right), 171.57$ $\left(\mathrm{CH}_{2} \mathrm{CO}_{2} \mathrm{CH}=\right), 170.39,170.15,169.95,169.44\left(\mathrm{CH}_{3} \mathrm{CO}_{2}\right), 168.70$ $\left(\equiv \mathrm{CNHCOCH}_{2}\right), 139.56(\mathrm{C}-5$ von $\mathrm{Chol}), 122.57$ (C-6 von Chol), $101.29\left(\mathrm{C}-1^{\prime}\right), 74.24$ (C-3 von $\left.\mathrm{Chol}\right), 70.76\left(\mathrm{C}-5^{\prime}\right), 70.47\left(\mathrm{C}-3^{\prime}\right), 68.97$ $\left(\mathrm{C}-2^{\prime}\right), 68.13\left(\mathrm{OCH}_{2} \mathrm{C} \equiv\right), 66.89\left(\mathrm{C}^{\prime} 4^{\prime}\right), 61.06\left(\mathrm{C}-6^{\prime}\right), 59.17(\mathrm{C} \equiv), 56.60$ (C-14 von Chol), 56.02 (C-17 von $\mathrm{Chol}$ ), 49.92 (C-9 von $\mathrm{Chol}$ ), 42.81 ( $\mathrm{NHCOCH}_{2} \mathrm{CO}$ ), 42.23 (C-13 von Chol), 39.63 (C-16 von Chol), 39.44 (C-24 von Chol), 37.99 (C-4 von Chol), 36.89 (C-1 von Chol), 36.51 (C-10 von Chol), 36.10 (C-22 von Chol), 35.72 (C-20 von Chol), 31.83 (C-12 von Chol), 31.76 (C-8 von $\mathrm{Chol}$ ), 31.52 (C-7 von $\mathrm{Chol}$ ), $30.58\left(\mathrm{COCH}_{2} \mathrm{CH}_{2} \mathrm{CO}\right), 29.59\left(\mathrm{COCH}_{2} \mathrm{CH}_{2} \mathrm{CO}\right), 28.17(\mathrm{C}-2$ von Chol), 27.94 (C-25 von $\mathrm{Chol}$ ), 24.21 (C-15 von $\mathrm{Chol}$ ), 23.74 (C-23 von Chol), 22.78 (C-27 von Chol), 22.51 (C-26 von Chol), 20.96 (C11 von $\mathrm{Chol}$ ), 20.76, 20.67, 20.62, $20.52\left(\mathrm{CH}_{3} \mathrm{CO}\right), 19.25$ (C-19 von Chol), 18.65 (C-21 von Chol), 11.80 (C-18 von Chol). - FAB-MS: $m / z(\%)=1637(2)\left[\mathrm{M}+\mathrm{H}^{+}\right], 1307$ (2), 1289 (21), $1112(17), 1070$ (1.5), 1052 (1.5), 921 (14), 782 (2.5), 750 (2), 591 (6), 331 (100).

$$
\begin{array}{ll}
\mathrm{C}_{79} \mathrm{H}_{116} \mathrm{~N}_{2} \mathrm{O}_{34} \cdot 2 \mathrm{H}_{2} \mathrm{O}(1673.8) & \text { Ber. C } 56.69 \mathrm{H} 7.23 \mathrm{~N} 1.67 \\
& \text { Gef. C } 56.87 \mathrm{H} 7.29 \mathrm{~N} 1.36
\end{array}
$$

9) $N^{\alpha}-[4-(5-C h o l e s t e n-3 \beta-y l o x y)$ succinyl $]-N$ - $[$ tris $(\beta$-D-galactopyranosyloxymethyl) methyl /glycinamid $\left[(\mathrm{Gal})_{3}\right.$-TRIS-Gly-SUCCChol, 6b] (vgl. Lit. $\left.{ }^{9}\right)$ : $\mathrm{Zu}$ einer auf $-20^{\circ} \mathrm{C}$ gekühlten, gut gerührten Lösung von $1.47 \mathrm{~g}(0.89 \mathrm{mmol}) \mathbf{6} \mathbf{a}$ in $147 \mathrm{ml} \mathrm{MeOH}$ gibt man aus einer Spritze unter Argon langsam $0.74 \mathrm{ml}(743 \mathrm{mg}, 23.2 \mathrm{mmol}$ ) wasserfreies Hydrazin zu. Nach weiterem 6 stdg. Rühren bei $-20^{\circ} \mathrm{C}$ läßt man $5 \mathrm{~d}$ bei $-20^{\circ} \mathrm{C}$ stehen. Danach wird mit $1 \mathrm{ml}$ Aceton versetzt und zur Trockne eingedampft. Der sirupöse Rückstand wird mit $0.2 \mathrm{ml} \mathrm{MeOH}$ und $10 \mathrm{ml}$ Pentan vermischt. Der Niederschlag wird abzentrifugiert und mittels SC [Chloroform/ $/ \mathrm{MeOH} /$ Wasser (54:40:6)] aufgetrennt. Man erhält zwei Fraktionen. Die erste liefert nach Trocknen über $\mathrm{P}_{4} \mathrm{O}_{10}$ 6b. - Ausb. $340 \mathrm{mg} \mathrm{(33 \% ),}$ Schmp. $220^{\circ} \mathrm{C}$ (Zers.). - DC-C: $R_{\mathrm{f}}=0.21 .-[\alpha]_{\mathrm{D}}^{20}=-12.2(c=$ 0.5 in DMSO) (Lit. ${ }^{9)}:[\alpha]_{\mathrm{D}}^{20}=-9.7\left[c=1\right.$ in $\mathrm{CHCl}_{3} / \mathrm{DMSO}$ (1:1)]. $-{ }^{1} \mathrm{H}-\mathrm{NMR}\left(400 \mathrm{MHz}, \mathrm{CDCl}_{3} /\left[\mathrm{D}_{6}\right] \mathrm{DMSO}\right): \delta=8.02(\mathrm{t}$ $J=3.5 \mathrm{~Hz}, 1 \mathrm{H}, \mathrm{NH}), 7.07(\mathrm{~s}, 1 \mathrm{H}, \mathrm{NH}), 5.3(\mathrm{~m}, 1 \mathrm{H}, 6-\mathrm{H}$ von $\mathrm{Chol})$ 4.85 (br. s, $3 \mathrm{H}, \mathrm{OH}$ ), 4.65 (br. s, $3 \mathrm{H}, \mathrm{OH}$ ), 4.55 (br. s, $3 \mathrm{H}, \mathrm{OH}$ ), 4.40 $(\mathrm{m}, 1 \mathrm{H}, 3-\mathrm{H}$ von Chol), 4.36 (br. s, $3 \mathrm{H}, \mathrm{OH}), 4.13$ (d, $J_{1,2}=7.5$ $\left.\mathrm{Hz}, 3 \mathrm{H}, 1^{\prime}-\mathrm{H}\right), 4.07\left(\mathrm{~d}, J=10.5 \mathrm{~Hz}, 3 \mathrm{H}, \equiv \mathrm{CC} H_{\mathrm{A}} \mathrm{HO}\right), 3.78(\mathrm{~d}, J=$ $\left.10.5 \mathrm{~Hz}, 3 \mathrm{H}, \equiv \mathrm{CCH}_{\mathrm{B}} \mathrm{HO}\right), 3.66\left(\mathrm{~m}, 5 \mathrm{H}, 4^{\prime}-\mathrm{H}\right.$ und $\left.\mathrm{COCH}_{2} \mathrm{NH}\right), 3.53$ $\left(\mathrm{m}, 6 \mathrm{H}, 6^{\prime}-\mathrm{H}\right), 3.33\left(\mathrm{~m}, 9 \mathrm{H}, 2^{\prime}-, 3^{\prime}-\right.$ und $\left.5^{\prime}-\mathrm{H}\right), 2.48(\mathrm{~m}, 4 \mathrm{H}$, $\left.\mathrm{COCH}_{2} \mathrm{CH}_{2} \mathrm{CO}\right), 2.25-0.63(43 \mathrm{H}) .-{ }^{13} \mathrm{C}-\mathrm{NMR}(100 \mathrm{MHz}$, $\left.\mathrm{CDCl}_{3} /\left[\mathrm{D}_{6}\right] \mathrm{DMSO}\right): \delta=171.53,171.25,168.98,139.27,121.94$ $104.23,75.04,73.24,73.16,70.69,68.20,67.46,60.48,59.50,56.06$, $55.46,49.35,42.47,41.74,37.58,36.42,36.00,35.56,35.12,31.27$ $29.68,29.13,27.68,27.34,27.23,23.75,23.13,22.52,22.25,20.46$ 18.86, 18.37, 11.51. - FAB-MS: $m / z(\%)=1155(5)\left[\mathrm{M}+\mathrm{Na}^{+}\right]$, 
$1133(20)\left[\mathrm{M}+\mathrm{H}^{+}\right], 1079(2), 1061(2), 971(6), 953(6), 899(1), 809$ (3), 791 (3), 737 (1), 369 (100), 279 (36), 261 (65).

$\mathrm{C}_{55} \mathrm{H}_{92} \mathrm{~N}_{2} \mathrm{O}_{22} \cdot 3 \mathrm{H}_{2} \mathrm{O}(1187.4)$ Ber. C $55.64 \mathrm{H} 8.32 \mathrm{~N} 2.36$ Gef. C 55.63 H 8.10 N 2.45

Aus der zweiten Fraktion isoliert man die Monoacetyl-Verbindung 6c. - Ausb. $274 \mathrm{mg}(25 \%)$, Schmp. $260^{\circ} \mathrm{C}$ (Zers.). - DC-C: $R_{\mathrm{f}}=$ 0.41. - FAB-MS: $m / z=1197\left[\mathrm{M}+\mathrm{Na}^{+}\right]$.

Die Abspaltung der Acetyl-Gruppe erfolgt ähnlich wie voranstehend für 6a beschrieben: $\mathrm{Zu} 200 \mathrm{mg} \mathrm{6c}(0.17 \mathrm{mmol})$ in $5 \mathrm{ml} \mathrm{absol}$. $\mathrm{MeOH}$ gibt man bei $-20^{\circ} \mathrm{C} 5 \mu 1(5.1 \mathrm{mg}, 0.16 \mathrm{mmol})$ Hydrazin. Nach 3 tägigen Stehenlassen bei $-20^{\circ} \mathrm{C}$ arbeitet man wie voranstehend (Abschnitt 9) beschrieben auf. SC [Chloroform/ $\mathrm{MeOH} /$ Wasser (54:40:6)] liefert 6b, dessen Eigenschaften (Schmp., $\boldsymbol{R}_{\mathrm{f}}$ ) mit denen des zuvor aus $\mathbf{6} \mathbf{a}$ erhaltenen $\mathbf{6} \mathbf{b}$ übereinstimmen. - Ausb. $87 \mathrm{mg}(45 \%)$.

10) $N^{x}$-[4-(5-Cholesten-3 $\beta$-yloxycarbonylmethylamino $)$ succinyl $]$ $N$-[tris (2,3,4,6-tetra-O-acetyl- $\beta$ - $D$-galactopyranosyloxymethyl)methyl Jglycinamid $\left[\left(\mathrm{Ac}_{4} \mathrm{Gal}\right)_{3}\right.$-TRIS-Gly-SUCC-Gly-O-Chol, 6d]: $\mathrm{Zu}$ einer Lösung von $440 \mathrm{mg}(0.33 \mathrm{mmol}) \mathbf{3 f}$ und $222 \mathrm{mg}(0.40$ mmol) $5 \mathbf{d}$ (Hydrotrifluoracetat) in $50 \mathrm{ml}$ Dichlormethan gibt man $0.1 \mathrm{ml}(0.5 \mathrm{mmol}) \mathrm{N}$-Ethyldiisopropylamin und $130 \mathrm{mg}(0.5 \mathrm{mmol})$ EEDQ. Nach 24 stdg. Rühren bei $20^{\circ} \mathrm{C}$ wird eingedampft und der Rückstand mit Pentan vermischt. Das farblose Produkt wird mittels $\mathrm{SC}$ [Toluol/EtOH (9:1)] gereinigt und über $\mathrm{P}_{4} \mathrm{O}_{10}$ getrocknet. Ausb. $0.54 \mathrm{~g}(94 \%)$, Schmp. $127-130^{\circ} \mathrm{C}$. - DC-G: $R_{\mathrm{f}}=0.28$. $[\alpha]_{\mathrm{D}}^{20}=-22.9\left(c=1\right.$ in $\left.\mathrm{CHCl}_{3}\right) .-{ }^{1} \mathrm{H}-\mathrm{NMR}\left(400 \mathrm{MHz}, \mathrm{CDCl}_{3}\right)$ : $\delta=6.76(\mathrm{t}, J=5.0 \mathrm{~Hz}, 1 \mathrm{H}, \mathrm{NH}), 6.72(\mathrm{t}, J=5.2 \mathrm{~Hz}, 1 \mathrm{H}, \mathrm{NH})$, $6.26(\mathrm{~s}, 1 \mathrm{H}, \mathrm{NH}), 5.31\left(\mathrm{dd}, J_{3^{\prime}, 4^{\prime}}=3.2 \mathrm{~Hz}, J_{4^{\prime}, 5^{\prime}}=1.1 \mathrm{~Hz}, 3 \mathrm{H}\right.$, $\left.4^{\prime}-\mathrm{H}\right), 5.29\left(\mathrm{~m}, 1 \mathrm{H}, 6-\mathrm{H}\right.$ von Chol), $5.04\left(\mathrm{dd}, J_{2^{\prime}, 3^{\prime}}=10.3 \mathrm{~Hz}, J_{1^{\prime}, 2^{\prime}}=\right.$ $\left.7.5 \mathrm{~Hz}, 3 \mathrm{H}, 2^{\prime}-\mathrm{H}\right), 4.97\left(\mathrm{dd}, J_{2^{\prime}, 3^{\circ}}=10.3 \mathrm{~Hz}, J_{3^{\prime}, 4^{\prime}}=3.4 \mathrm{~Hz}, 3 \mathrm{H}\right.$, $\left.3^{\prime}-\mathrm{H}\right), 4.58\left(\mathrm{~m}, 1 \mathrm{H}, 3-\mathrm{H}\right.$ von Chol), $4.38\left(\mathrm{~d}, J_{1^{\prime}, 2^{\prime}}=7.5 \mathrm{~Hz}, 3 \mathrm{H}\right.$, $\left.1^{\prime}-\mathrm{H}\right), 4.12\left(\mathrm{dd}, J_{\text {gem. }}=10.4 \mathrm{~Hz}, J_{5 ; 6 \mathrm{~A}^{\prime}}=6.5 \mathrm{~Hz}, 6^{\prime}-\mathrm{H}_{\mathrm{A}}\right), 4.08(\mathrm{~d}$, $\left.J=10.3 \mathrm{~Hz}, \equiv \mathrm{CC} H_{\mathrm{A}} \mathrm{HO}\right), 4.05\left(\mathrm{dd}, J_{\mathrm{gem} .}=10.4 \mathrm{~Hz}, J_{5^{\prime}, 6 \mathbf{B}^{\prime}}=6.5\right.$ $\mathrm{Hz}, 6^{\prime}-\mathrm{H}_{\mathrm{B}}, 9 \mathrm{H}$ zusammen mit den Signalen bei $\delta=4.12$ und 4.08), 3.91 (t-artiges $\mathrm{m}, J_{5 ; 6^{\prime}}=6.5 \mathrm{~Hz}, 5 \mathrm{H}, 5^{\prime}-\mathrm{H}$ und $\mathrm{NHCH}_{2} \mathrm{CO}$ ), 3.78 (br. d, $\left.J=5.1 \mathrm{~Hz}, 2 \mathrm{H}, \mathrm{NHCH}_{2} \mathrm{CO}\right), 3.69$ (d, $J=10.3 \mathrm{~Hz}, 3 \mathrm{H}$, $\equiv \mathrm{CCH} H_{\mathrm{B}} \mathrm{HO}$ ), 2.55 (s, $\left.4 \mathrm{H}, \mathrm{COCH}_{2} \mathrm{CH}_{2} \mathrm{CO}\right), 2.4-0.6[\mathrm{~m}$ mit s bei $\delta=2.08,1.99,1.98,1.89,0.94$ und 0.60 sowie $\mathrm{d}$ bei $\delta=0.74(\mathrm{~J}=$ $7.0 \mathrm{~Hz}), 0.69(J=7.0 \mathrm{~Hz})$ und $0.68(J=7.0 \mathrm{~Hz}), 79 \mathrm{H}, \mathrm{CH}_{3} \mathrm{CO}$ und $\mathrm{H}$ von Chol]. - ${ }^{13} \mathrm{C}-\mathrm{NMR}\left(50.3 \mathrm{MHz}, \mathrm{CDCl}_{3}\right): \delta=172.37$, $172.29,170.38,170.15,169.97,169.48,169.35,169.05,139.22,122.85$, $101.27,75.19,70.68,70.47,68.99,68.02,66.92,61.08,59.33,56.57$, $56.01,49.88,43.13,42.20,41.52,39.60,39.40,37.88,36.79,36.51$, $36.07,35.68,31.79,31.72,31.06,30.1,28.13,27.91,24.17,23.71,22.74$, $22.48,20.92,20.73,20.63,20.58,20.47,19.19,18.62,11.76$.

$\mathrm{C}_{81} \mathrm{H}_{11} \mathrm{~N}_{3} \mathrm{O}_{35} \cdot 3 \mathrm{H}_{2} \mathrm{O}(1748.9)$ Ber. C $55.63 \mathrm{H} 7.20 \mathrm{~N} 2.40$ Gef. C 55.93 H 7.13 N 1.87

11) $N^{\alpha}-[4-(5-$ Cholesten-3 $\beta-y$ loxycarbonylmethylamino $)-N$ [tris( $\beta$-D-galactopyranosyloxymethyl) methyl]succinyl]glycinamid $\left[(\mathrm{Gal})_{3}\right.$-TRIS-Gly-SUCC-Gly-O-Chol, 6e $]$ : $\mathrm{Zu}$ einer auf $-20^{\circ} \mathrm{C}$ gekühlten Lösung von $316 \mathrm{mg}(0.18 \mathrm{mmol}) \mathbf{6 d}$ in $20 \mathrm{ml} \mathrm{MeOH}$ werden unter starkem Rühren langsam $75 \mu \mathrm{l}(75.8 \mathrm{mg}, 2.37 \mathrm{mmol})$ wasserfreies Hydrazin gegeben. Man rührt $6 \mathrm{~h}$ bei $-20^{\circ} \mathrm{C}$ und läßt dann $24 \mathrm{~h}$ bei $-20^{\circ} \mathrm{C}$ und $72 \mathrm{~h}$ bei $20^{\circ} \mathrm{C}$ stehen und arbeitet wie in Abschnitt 9 beschrieben auf. - Ausb. $80 \mathrm{mg}$ (36\%), Schmp. $220^{\circ} \mathrm{C}$ (Zers). - DC-C: $R_{\mathrm{f}}=0.17 .-[\alpha]_{\mathrm{D}}^{20}=-14.8(c=0.25$ in DMSO). $-{ }^{1} \mathrm{H}-\mathrm{NMR}\left(200 \mathrm{MHz},\left[\mathrm{D}_{6}\right] \mathrm{DMSO}\right): \delta=8.29(\mathrm{~s}, 1 \mathrm{H}$, NH), 8.06 (s, $1 \mathrm{H}, \mathrm{NH}), 7.05$ (s, $1 \mathrm{H}, \mathrm{NH}), 5.35$ (br. s, $1 \mathrm{H}, 6-\mathrm{H}$ von Chol), 4.89 (br. s, $3 \mathrm{H}, \mathrm{OH}$ ), 4.75 (br. s, $3 \mathrm{H}, \mathrm{OH}$ ), 4.63 (br. s, $3 \mathrm{H}$, $\mathrm{OH}), 4.41(\mathrm{~m}, 4 \mathrm{H}, 3-\mathrm{H}$ von $\mathrm{Chol}$ und $\mathrm{OH}), 4.20-3.95\left(\mathrm{~m}, 6 \mathrm{H}, 1^{\prime}\right.$ $\mathrm{H}$ und $\left.\equiv \mathrm{CCH}_{\mathrm{A}} \mathrm{HO}\right), 3.76\left(\mathrm{~s}, 3 \mathrm{H}, \equiv \mathrm{CCH}_{\mathrm{B}} \mathrm{HO}\right), 3.63\left(\mathrm{~m}, 7 \mathrm{H}, 4^{\prime}-\mathrm{H}\right.$ und $\left.\mathrm{NHCH}_{2} \mathrm{CO}\right), 3.48\left(\mathrm{~s}, 6^{\prime}-\mathrm{H}\right), 3.4-3.2\left(\mathrm{~m}, 9 \mathrm{H}, 2^{\prime}-3^{\prime}-\right.$ und $\left.5^{\prime}-\mathrm{H}\right)$,
2.37 (s, $\left.4 \mathrm{H}, \mathrm{COCH}_{2} \mathrm{CH}_{2} \mathrm{CO}\right), 2.3-0.6(\mathrm{~m}, 43 \mathrm{H}) .-{ }^{13} \mathrm{C}-\mathrm{NMR}(100$ MHz, [D $]$ DMSO): $\delta=172.24,172.02,169.14,169.03,138.96$, $122.10,104.04,74.93,73.87,73.16,70.69,68.33,67.53,60.69,59.33$, $56.02,55.42,49.30,42.88,41.69,40.69,39.09,38.81,37.51,36.32$, $35.95,35.53,35.09,31.26,31.21,30.51,30.40,27.64,27.32,27.16$, $23.70,23.12,22.44,22.17,20.41,18.79,18.29,11.43$. - FAB-MS: $m / z(\%)=1212(1)\left[\mathrm{M}+\mathrm{Na}^{-1}\right], 1028(0.4), 1010(1), 866(1), 848$ (2), 794 (3), 704 (4), 686 (6), 608 (2), 369 (100).

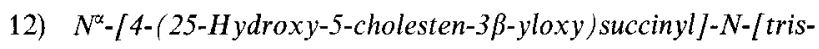
(2,3,4,6-tetra-O-acetyl- $\beta$-D-galactopyranosyloxymethyl)methyl $/ g l y$ cinamid [( $\mathrm{Ac}_{4} \mathrm{Gal}_{3}$-TRIS-Gly-SUCC-25-OH-Chol, 6f]: $\mathrm{Zu}$ einer Lösung von $150 \mathrm{mg}(0.11 \mathrm{mmol}) 3 \mathrm{f}$ in $2 \mathrm{ml}$ Dichlormethan gibt man $41 \mathrm{mg}(0.125 \mathrm{mmol}) 4$ und $10 \mu \mathrm{l}(9.8 \mathrm{mg}, 0.12 \mathrm{mmol})$ Pyridin. Nach 18stdg. Rühren bei $20^{\circ} \mathrm{C}$ wird mit $12 \mathrm{mg}(0.03 \mathrm{mmol}) 25-$ Hydroxy-5-cholesten- $3 \beta$-ol und $10 \mu \mathrm{l}(7.4 \mathrm{mg}, 60 \mu \mathrm{mol}) \mathrm{N}$-Ethyldiisopropylamin versetzt und $20 \mathrm{~h}$ bei $20^{\circ} \mathrm{C}$ gerührt. Anschließend wäscht man mehrmals mit 20 proz. Citronensäure-Lösung, ges. Natriumhydrogencarbonat-Lösung und Wasser (vgl. Abschnitt 5), trocknet mit $\mathrm{MgSO}_{4}$ und dampft zur Trockne ein. Der Rückstand wird mittels SC $\left[\mathrm{CHCl}_{3} /\right.$ Aceton (3:1)] gereinigt und anschließend über $\mathrm{P}_{4} \mathrm{O}_{10}$ getrocknet. - Ausb. $27 \mathrm{mg} \mathrm{(53 \% ),} \mathrm{Schmp.}$ $115-118^{\circ} \mathrm{C}$. DC-H: $R_{\mathrm{f}}=0.34 .-[\alpha]_{\mathrm{D}}^{20}=-24.7(c=1$ in $\left.\mathrm{CHCl}_{3}\right) .-{ }^{1} \mathrm{H}-\mathrm{NMR}\left(200 \mathrm{MHz}, \mathrm{CDCl}_{3}\right): \delta=6.50(\mathrm{t}, J=4.5 \mathrm{~Hz}$, $1 \mathrm{H}, \mathrm{NH}), 6.17(\mathrm{~s}, 1 \mathrm{H}, \mathrm{NH}), 5.32\left(\mathrm{dd}, J_{3^{\prime}, 4^{\prime}}=3.3 \mathrm{~Hz}, J_{4^{\prime} 5^{\prime}}=1.0 \mathrm{~Hz}\right.$, $\left.3 \mathrm{H}, 4^{\prime}-\mathrm{H}\right), 5.18(\mathrm{~m}, 1 \mathrm{H}, 6-\mathrm{H}$ von $25-\mathrm{HO}-\mathrm{Chol}), 5.07$ (dd, $J_{2^{\prime}, 3^{\prime}}=$ $\left.10.5 \mathrm{~Hz}, J_{1^{\prime}, 2^{\prime}}=7.7 \mathrm{~Hz}, 3 \mathrm{H}, 2^{\prime}-\mathrm{H}\right), 4.96\left(\mathrm{dd}, J_{2^{\prime}, 3^{\prime}}=10.5 \mathrm{~Hz}, J_{3^{\prime}, 4^{\prime}}=\right.$ $3.3 \mathrm{~Hz}, 3 \mathrm{H}, 3^{\prime}-\mathrm{H}$ ), 4.53 (br. m, $1 \mathrm{H}, 3-\mathrm{H}$ von $25-\mathrm{HO}-\mathrm{Chol}$ ), 4.37 (d, $\left.J=7.7 \mathrm{~Hz}, 3 \mathrm{H}, 1^{\prime}-\mathrm{H}\right), 4.18-4.02\left(\mathrm{~m}, 9 \mathrm{H}, 6^{\prime}-\mathrm{H}\right.$ und $\left.\equiv \mathrm{CCH}_{\mathrm{A}} \mathrm{HO}\right)$, $3.95-3.75\left(\mathrm{~m}, 5 \mathrm{H}, 5^{\prime}-\mathrm{H}\right.$ und $\left.\mathrm{NHCH}_{2} \mathrm{CO}\right), 3.70(\mathrm{~d}, J=10.5 \mathrm{~Hz}$, $2 \mathrm{H}$, $\equiv \mathrm{CCH}_{\mathrm{B}} \mathrm{HO}$ ), $2.62-2.47\left(\mathrm{~m}, 4 \mathrm{H}, \mathrm{COCH}_{2} \mathrm{CH}_{2} \mathrm{CO}\right.$ ), 2.24 (br. d, $J=7.5 \mathrm{~Hz}, 2 \mathrm{H}, 4-\mathrm{H}$ von $25-\mathrm{HO}-\mathrm{Chol}$ ), $2.17-0.9$ (m mit s bei $\delta=$ $2.10,2.02,1.93,1.13,0.94,70 \mathrm{H}, \mathrm{CH}_{3} \mathrm{CO}$ und $\mathrm{H}$ von $25-\mathrm{HO}-\mathrm{Chol}$ ), $0.87(\mathrm{~d}, J=6.7 \mathrm{~Hz}, 3 \mathrm{H}, 21-\mathrm{H}$ von $25-\mathrm{HO}-\mathrm{Chol}), 0.61$ (s, $3 \mathrm{H}, 19$ $\mathrm{H}$ von 25 -HO-Chol). $-{ }^{13} \mathrm{C}-\mathrm{NMR}\left(50.3 \mathrm{MHz}, \mathrm{CDCl}_{3}\right): \delta=172.21$, 171.60, 170.41, 170.17, 169.97, 169.46, 168.73, 139.56, 122.56, 101.28, $74.23,70.96,70.74,70.46,68.96,68.10,66.88,61.04,59.18,56.58$, $55.97,49.89,44.34,42.80,42.24,39.62,37.98,36.88,36.50,36.37$, $35.70,31.81,31.75,30.56,29.58,29.25,29.11,28.18,27.64,24.20$, $20.94,20.76,20.67,20.62,20.52,19.24,18.61,11.79$. - FAB-MS: $m / z(\%)=1676(8)\left[\mathrm{M}+\mathrm{Na}^{+}\right], 1654(4)\left[\mathrm{M}+\mathrm{H}^{+}\right], 1323(3), 1305$ (23), $1169(2), 1112(31), 1070(9), 1052$ (27), 921 (25), 782 (12), 750 (12), $367(12), 331(73), 109(100)$.

\section{$\mathrm{C}_{79} \mathrm{H}_{116} \mathrm{~N}_{2} \mathrm{O}_{35} \cdot 2 \mathrm{H}_{2} \mathrm{O}(1689.8)$ Ber. C $56.15 \mathrm{H} 7.16 \mathrm{~N} 1.66$ Gef. C 56.42 H 7.15 N 1.66}

13) $N^{x}$ - [4-(25-Hydroxy-5-cholesten-3B-yloxy) succinyl]- $N$-[tris$(\beta$-D-galactopyranosyloxymethyl)methyl $]$ glycinamid $\left[(\mathrm{Gal})_{3}\right.$-TRISGly-SUCC-25-HO-Chol, 6g]: $\mathrm{Zu}$ einer Lösung von $20 \mathrm{mg}(12$ $\mu \mathrm{mol}$ ) $6 \mathrm{f}$ in $5 \mathrm{ml} \mathrm{MeOH}$ gibt man aus einer Spritze bei $0^{\circ} \mathrm{C} 30 \mu \mathrm{l}$ (30.3 mg, $0.95 \mathrm{mmol}$ ) wasserfreies Hydrazin. Nach 4 tägigem Stehenlassen bei $-20^{\circ} \mathrm{C}$ wird zweimal nacheinander mit je $2 \mathrm{ml}$ Aceton versetzt und eingedampft. Man reinigt den farblosen Rückstand mittels SC $\left[\mathrm{CHCl}_{3} / \mathrm{MeOH} /\right.$ Wasser (54:40:6)] und erhält farbloses, glasartiges 6g. - Ausb. $5.0 \mathrm{mg}(36 \%)$ ) - DC-C: $R_{\mathrm{f}}=0.48$. $[\alpha]_{\mathrm{D}}^{20}=-10.12\left(c=1\right.$ in DMSO). - ${ }^{1} \mathrm{H}-\mathrm{NMR}(400 \mathrm{MHz}$, [D $\mathrm{D}_{6}$ DMSO): $\delta=8.07(\mathrm{t}, J=5.5 \mathrm{~Hz}, 1 \mathrm{H}, \mathrm{NH}), 7.08(\mathrm{~s}, 1 \mathrm{H}, \mathrm{NH})$, $5.34(\mathrm{~m}, 1 \mathrm{H}, 6-\mathrm{H}$ von $25-\mathrm{HO}-\mathrm{Chol}), 4.91$ (d, $J=3.8 \mathrm{~Hz}, 3 \mathrm{H}, \mathrm{OH})$, 4.77 (br. s, $3 \mathrm{H}, \mathrm{OH}), 4.64$ (t, $J=5.5 \mathrm{~Hz}, 3 \mathrm{H}, \mathrm{OH}), 4.43$ (m mit d, $J=4.8 \mathrm{~Hz}, 4 \mathrm{H}$, OH und $3-\mathrm{H}$ von $25-\mathrm{HO}-\mathrm{Chol}), 4.16-4.01[\mathrm{~m}$ mit d bei $\delta=4.11, J=7.0 \mathrm{~Hz}$, s bei $\delta=4.06$ und $\mathrm{d}$ bei $\delta=4.04$ $(J=10.5 \mathrm{~Hz}), 10 \mathrm{H}, 1^{\prime}-\mathrm{H}, \equiv \mathrm{CCH}_{\mathrm{A}} \mathrm{HO}$ und $\mathrm{OH}$ von $\left.25-\mathrm{HO}-\mathrm{Chol}\right]$, $3.73\left(\mathrm{~d}, J=10.5 \mathrm{~Hz}, 3 \mathrm{H}, \equiv \mathrm{CCH}_{\mathrm{B}} \mathrm{HO}\right), 3.67(\mathrm{~d}, J=5.5 \mathrm{~Hz}, 2 \mathrm{H}$, $\left.\mathrm{NHCH}_{2} \mathrm{CO}\right), 3.61$ (t-artiges $\left.\mathrm{m}, 3 \mathrm{H}, 4^{\prime}-\mathrm{H}\right), 3.56-3.42\left(\mathrm{~m}, 6 \mathrm{H}, 6^{\prime}-\mathrm{H}\right)$, $3.42-3.15\left(\mathrm{~m}, 9 \mathrm{H}, 2^{\prime}-, 3^{\prime}-\right.$ und $\left.5^{\prime}-\mathrm{H}\right), 2.47-2.39(\mathrm{~m}, 4 \mathrm{H}$, 
$\mathrm{COCH}_{2} \mathrm{CH}_{2} \mathrm{CO}$ ), 2.25 (d, $J=8.0 \mathrm{~Hz}, 2 \mathrm{H}, 4-\mathrm{H}$ von $25-\mathrm{HO}-\mathrm{Chol}$ ), $2.1-0.9[\mathrm{~m}$ mit s bei $\delta=1.02,0.98$ und $\mathrm{d}$ bei $\delta=0.90(J=6.5$ $\mathrm{Hz}$ ), $37 \mathrm{H}, \mathrm{H}$ von $25-\mathrm{HO}-\mathrm{Chol}$, 0.65 (s, $3 \mathrm{H}, \mathrm{H}$ von $25-\mathrm{HO}-\mathrm{Chol}$ ). ${ }^{13} \mathrm{C}-\mathrm{NMR}\left(100 \mathrm{MHz},\left[\mathrm{D}_{6}\right] \mathrm{DMSO}\right): \delta=171.81,171.22,169.02$, $139.59,122.08,104.49,75.18,73.31,73.27,70.73,68.80,68.16,67.38$, $60.34,59.68,56.15,55.62,49.44,48.62,44.16,42.37,41.88,39.20$, $37.66,36.51,36.21,36.12,35.26,31.39,31.30,29.70,29.43,29.25$, $29.19,27.83,27.34,23.90,20.59,20.27,19.01,18.59,11.71$. FABMS: $m / z(\%)=1171(6)\left[\mathrm{M}+\mathrm{Na}^{+}\right], 1149(4)\left[\mathrm{M}+\mathrm{H}^{+}\right], 987(2)$, 969 (2), 807, (2), 753 (2), 691 (4), 663 (2), 645 (3), 551 (5), 423 (2), 385 (3), 367 (22), 279 (10), 261 (19), 239 (13), 237 (10), 217 (40), 215 (14), 181 (17), 131 (48), 109 (37), 93 (20), 91 (100).

\section{CAS-Registry-Nummern}

1b: $29022-11-5$ / 2b: 127619-22-1 / 3c: $127619-23-2$ / 3e: $127619-$ 24-3 / 3f: 127645-43-6/3g: 127619-25-4 / 4: 54714-11-3/5a: 1510 21-0 / 5b: $127619-26-5 / 5$ c: $73670-11-8 /$ 5d: $73670-26-5 / 5 e: 2140-$ 46-7 /6a: 127619-21-0 / 6b: 91202-79-8 / 6c: 127619-27-6/6d: 127645-28-7 / 6e: 127619-28-7 / 6f: 127619-29-8 / 6g: 12761930-1 / TRIS: 77-86-1 / Boc-Gly-O-TDO: 69668-06-0 / Acetobromgalactose: $3068-32-4$ / Cholesterin: $57-88-5$

${ }^{1)}$ G. Ashwell, J. Harford, Annu. Rev. Biochem. 51 (1982) 531.

2) G. Barratt, J.-P. Tenu, A. Yapo, J.-F. Petet, Biochim. Biophys. Acta 862 (1986) 153.

3) L. Fiume, B. Bassi, C. Busi, A. Mattioli, G. Spinosa, H. Faulstich, FEBS Lett. 203 (1986) 203.

4) P. R. Dragsten, D. B. Mitchell, G. Covert, T. Baker, Biochim. Biophys. Acta 926 (1987) 270.

5) A. D. Attie, R. C. Pittman, D. Steinberg, Proc. Natl. Acad. Sci. 77 (1980) 5923

6) J. Hartford, G. Ashwell, Glycoconiugates 4 (1982) 27.

7) R. T. Lee, P. Lin, Y. C. Lee, Biochemistry 23 (1984) 4255.

${ }^{8)}$ H. Lee, S. Kelm, T. Yoshino, R. Schauer, Biol. Chem. HoppeSeyler 369 (1988) 705.
${ }^{9)}$ H.-J. M. Kempen, C. Hoes, J. H. van Boom, H. H. Spanjer, J. de Lange, A. Langendoen, T. J. C. van Berkel, J. Med. Chem. 27 (1984) 1306.

10) H.-J. M. Kempen, F. Kuipers, T. J. C. van Berkel, R. J. Vonk, J. Lipid Res. 28 (1987) 659.

11) T. J. C. van Berkel, J. K. Kruit, H. H. Spanjer, J. F. Nagelkerke, L. Harkes, H.-J. M. Kempen, J. Biol. Chem. 260 (1985) 2694; T. J. C. van Berkel, J. K. Kruijt, H.-J. M. Kempen, J. Biol. Chem. 260 (1985) 12203.

12) Durchführung der Berechnungen mit dem Programm: M. J. C. Crabbe, J. R. Appleyard, Desktop Molecular Modeller, Oxford University Press, Oxford 1988.

13) B. Helferich, B. Zirner, Chem. Ber. 95 (1962) 2604

14) Y. C. Lee, Carbohydr. Res. 67 (1978) 509

15) G. Wulff, G. Röhle, Angew. Chem. 86 (1974) 173; Angew. Chem. Int. Ed. Engl. 13 (1974) 157; G. Wulf,, W. Schmidt, Carbohydr. Res. 53 (1977) 33.

16) E. Batyka, Mag. Biol. Kutatintez. Munkai 13 (1941) 334 [Chem. Abstr. 36 (1942) 484].

17) O. Hollitzer, A. Seewald, W. Steglich, Angew. Chem. 88 (1976) 480; Angew. Chem. Int. Ed. Engl. 15 (1976) 444.

18) W. Steglich, U. Hamacher, H. Rehwinkel, Publikation in Vorbereitung.

19) H. Kunz, S. Birnbach, Angew. Chem. 98 (1986) 354; Angew. Chem. Int. Ed. Engl. 25 (1986) 360; N. V. Bovin, S. E. Zurabyan, A. Y. Khorlin, J. Carbohydr. Chem. 2 (1983) 249; Y. Ishido, N. Sakairi, M. Sekiya, N. Nakazaki, Carbohydr. Res. 97 (1981) 51.

${ }^{20)}$ H. Egge, J. Peter-Katalinić, Mass Spectrom. Rev. 6 (1987) 331.

${ }^{21)}$ G. Schnorrenberg, W. Steglich, Angew. Chem. 91 (1979) 326; Angew. Chem. Int. Ed. Engl. 18 (1979) 307.

${ }^{22)}$ M. Fieser, L. F. Fieser, Reagents for Organic Synthesis, Bd. 2, S. 191, Wiley, New York 1969.

23) L. A. Carpino, G. Y. Han, J. Org. Chem. 37 (1972) 3403.

${ }^{24)}$ W. G. Salmond, M. C. Sobola, Tetrahedron Lett. 1977, 1695; J. J. Partridge, S. Faber, M. R. Uskoković, Helv. Chim. Acta 57 (1974) 764.

25) L. Lapatsanis, C. Profilis, P. Catsoulacos, J. Chem. Eng. Data 25 (1980) 287 [Chem. Abstr. 93 (1980) 11495n].

26) H. J. Reich, M. Jautelat, M. T. Messe, F. J. Weigert, J. D. Roberts, J. Am. Chem. Soc. 91 (1969) 7445.

[51/90] 